\title{
CNFE-SE: a novel approach combining complex network-based feature engineering and stacked ensemble to predict the success of intrauterine insemination and ranking the features
}

Sima Ranjbari ${ }^{1}$, Toktam Khatibi ${ }^{1 *}$ (D, Ahmad Vosough Dizaji ${ }^{2}$, Hesamoddin Sajadi ${ }^{4}$, Mehdi Totonchi ${ }^{3,4^{*}}$ and Firouzeh Ghaffari ${ }^{5}$

\begin{abstract}
Background: Intrauterine Insemination (IUI) outcome prediction is a challenging issue which the assisted reproductive technology (ART) practitioners are dealing with. Predicting the success or failure of IUI based on the couples' features can assist the physicians to make the appropriate decision for suggesting IUI to the couples or not and/or continuing the treatment or not for them. Many previous studies have been focused on predicting the in vitro fertilization (IVF) and intracytoplasmic sperm injection (ICSI) outcome using machine learning algorithms. But, to the best of our knowledge, a few studies have been focused on predicting the outcome of IUI. The main aim of this study is to propose an automatic classification and feature scoring method to predict intrauterine insemination (IUI) outcome and ranking the most significant features.
\end{abstract}

Methods: For this purpose, a novel approach combining complex network-based feature engineering and stacked ensemble (CNFE-SE) is proposed. Three complex networks are extracted considering the patients' data similarities. The feature engineering step is performed on the complex networks. The original feature set and/or the features engineered are fed to the proposed stacked ensemble to classify and predict IUI outcome for couples per IUI treatment cycle. Our study is a retrospective study of a 5-year couples' data undergoing IUI. Data is collected from Reproductive Biomedicine Research Center, Royan Institute describing 11,255 IUI treatment cycles for 8,360 couples. Our dataset includes the couples' demographic characteristics, historical data about the patients' diseases, the clinical diagnosis, the treatment plans and the prescribed drugs during the cycles, semen quality, laboratory tests and the clinical pregnancy outcome.

\footnotetext{
*Correspondence: toktam.khatibi@modares.ac.ir; m.totonchi@royaninstitute.

org

1 School of Industrial and Systems Engineering, Tarbiat Modares

University, Tehran, Iran

${ }^{3}$ Department of Reproductive Imaging, Reproductive Biomedicine

Research Center, Royan Institute for Reproductive Biomedicine, ACECR,

Tehran, Iran

Full list of author information is available at the end of the article
}

(C) The Author(s) 2021. Open Access This article is licensed under a Creative Commons Attribution 4.0 International License, which permits use, sharing, adaptation, distribution and reproduction in any medium or format, as long as you give appropriate credit to the original author(s) and the source, provide a link to the Creative Commons licence, and indicate if changes were made. The images or other third party material in this article are included in the article's Creative Commons licence, unless indicated otherwise in a credit line to the material. If material is not included in the article's Creative Commons licence and your intended use is not permitted by statutory regulation or exceeds the permitted use, you will need to obtain permission directly from the copyright holder. To view a copy of this licence, visit http://creativecommons.org/licenses/by/4.0/. The Creative Commons Public Domain Dedication waiver (http://creativeco mmons.org/publicdomain/zero/1.0/) applies to the data made available in this article, unless otherwise stated in a credit line to the data. 
Results: Experimental results show that the proposed method outperforms the compared methods with Area under receiver operating characteristics curve (AUC) of $0.84 \pm 0.01$, sensitivity of $0.79 \pm 0.01$, specificity of $0.91 \pm 0.01$, and accuracy of $0.85 \pm 0.01$ for the prediction of IUI outcome.

Conclusions: The most important predictors for predicting IUI outcome are semen parameters (sperm motility and concentration) as well as female body mass index (BMI).

Keywords: IUI outcome prediction, Complex networks, Feature engineering, Stacked ensemble classifier, Feature selection

\section{Background}

Infertility is defined as the failure of the female partner to conceive after at least one year of regular unprotected sexual intercourse [1]. More than 186 million people of the world's population specifically people living in developing countries are suffering from infertility [2]. In most cases, the causes of infertility are not clear, which complicates the treatment procedure. These problems have been exacerbated for several reasons, such as lifestyle changes, infection, and genetic issues. In many cases, the only way to get pregnant has been through the use of assisted reproductive technology (ART), and its performance has not yet been optimized [3].

Every year, more than 1.5 million ART cycles are carried out all over the world [4]. ART consists of three basic procedures including intrauterine insemination (IUI), in-vitro fertilization (IVF) and intracytoplasmic injection (ICSI) which are generally carried out in different steps of the treatment [5]. The first-line treatment, second and the third stages of ART are IUI, IVF, and ICSI, respectively [6]. In comparison with other sophisticated methods of ART, IUI has been considered as the easiest, minimally invasive and less expensive one. Most of the recent researches have shown the efficacy of IUI $[6,7]$.

IUI outcome prediction is a challenging issue which the ART practitioners are dealing with. Predicting the success or failure of IUI based on the couples' features can assist the physicians to make the appropriate decision for suggesting IUI to the couples or not and/or continuing the treatment or not for them [5].

Machine Learning approaches, as the modern scientific discipline, concentrates on how to detect the hidden patterns and extract the information from data. Machine learning provides different methods and algorithms to predict the output from some input predictors which can be used for clinical decision making [8].

To the best of our knowledge, many previous studies have been focused on predicting the IVF and ICSI outcome using machine learning methods as summarized in Table 1.

As illustrated by Table 2, the previous studies related to outcome prediction of ART methods are listed which have analyzed data using data mining and/or statistical methods. For this purpose, classifiers such as Decision Tree (DT), Logistic Regression (LR), Naïve Bayes (NB), K-Nearest Neighbors (K-NN), Support Vector Machines (SVM), Random Forest (RF), and Artificial Neural Networks (ANN) such as Multi-Layered Perceptron (MLP) and Radial Basis Function (RBF) have been used in the previous studies for predicting the clinical pregnancy after the complete cycles of different ART methods. A main drawback of the most of the considered previous studies is small volume of dataset and a few number of the considered features. Small dataset increases the risk of overfitting the trained models. Overfitting occurs when a model has good predictive ability for training dataset but shows poor performance for test dataset. Models with high overfitting property has lower generalization ability.

In this study, a dataset including the features of 11,255 IUI treatment cycles for 8360 couples is considered for IUI outcome prediction. Our dataset includes the couples' demographic characteristics, historical data about the patients' diseases, the clinical diagnosis, the treatment plans and the prescribed drugs during the cycles, semen quality, laboratory tests and the clinical pregnancy outcome. Considering the large number of couples and their corresponding IUI treatment cycles is a main advantage of this study compared to the considered previous studies.

On the other hand, most of the previous studies have considered the outcome prediction for IVF or ISCI. To the best of our knowledge, a few studies have been focused on predicting the outcome of IUI which have used clustering methods $[9,10]$ or regression analysis [11].

The previous studies which have been based on regression analysis only have considered the weights of the independent features to predict the overall pregnancy probability and they have not assessed the interconnection among the features [11-17]. Many previous studies have suffered from the lack of statistical power due to their small dataset $[17,18]$. Also, the AUC performance of the previously proposed models for predicting IUI outcome have been low [12]. Therefore, it is required to improve the prediction performance by proposing novel methods and considering more data records. 
Table 1 Summarizing the previous studies of predicting ART outcome

\begin{tabular}{|c|c|c|c|c|}
\hline Research problem & Dataset & Features & Analytical method & Remarks \\
\hline Predicting IVF outcomes & 5275 records & 67 different features & $\begin{array}{l}\text { Combination of Decision Tree } \\
\text { and Genetic algorithm }\end{array}$ & $\begin{array}{l}\text { Low predictive accuracy with } \\
73 \%\end{array}$ \\
\hline $\begin{array}{l}\text { Patient-specific predictions of } \\
\text { outcome after IUI }\end{array}$ & $\begin{array}{l}1438 \text { patients who underwer } \\
3375 \text { IUI cycles }\end{array}$ & to features & Logistic regression analysis & A few numbers of features \\
\hline $\begin{array}{l}\text { Predictive modeling of } \\
\text { implantation outcome in } \\
\text { IVF }\end{array}$ & 3898 embryos & 18 features & $\begin{array}{l}\text { Naive Bayes, Decision Tree, } \\
\text { K Nearest Neighbors, SVM, } \\
\text { multilayer perceptron, radial } \\
\text { basis function network }\end{array}$ & A small number of features \\
\hline $\begin{array}{l}\text { Determine the impact of } \\
\text { sperm morphology on the } \\
\text { success of IUI }\end{array}$ & $\begin{array}{l}412 \text { couples with } 530 \text { IUI } \\
\text { cycles }\end{array}$ & 12 features & statistical analysis & A few samples studied \\
\hline $\begin{array}{l}\text { Outcome prediction of IUI } \\
\text { based on sperm morphol- } \\
\text { ogy and progressively } \\
\text { motile sperm count }\end{array}$ & $\begin{array}{l}4251 \text { first IUI cycles of } 1166 \\
\text { couples }\end{array}$ & 9 features & $\begin{array}{l}\text { multivariable logistic regres- } \\
\text { sion }\end{array}$ & A few features considered \\
\hline $\begin{array}{l}\text { Predicting live birth after IVF } \\
\text { complete cycle }\end{array}$ & 113,873 women data & Age and duration of infertility & Logistic regression & $\begin{array}{l}\text { A few difference makers } \\
\text { considered }\end{array}$ \\
\hline $\begin{array}{l}\text { Identifying and choosing the } \\
\text { best sperms for ICSI }\end{array}$ & 219 patients & 13 features & $\begin{array}{l}\text { Naive Bayes, SVM, MLP, IBK, } \\
\text { K-Star, Random Committee, } \\
\text { J48, Random Forest }\end{array}$ & Small set of patients \\
\hline $\begin{array}{l}\text { IVF outcome prediction } \\
\text { relying on endometrial } \\
\text { transcriptions }\end{array}$ & 25 patients & 20 feature & PCA and HCA clustering & Small number of patients \\
\hline $\begin{array}{l}\text { Predicting Implantation Out- } \\
\text { come of IVF and ICSI }\end{array}$ & the data of 486 patients & 21 features & $\begin{array}{l}\text { SVM, Adaboost, RPART, RF, } \\
\text { 1-NN }\end{array}$ & A few features considered \\
\hline $\begin{array}{l}\text { Predicting the impact of } \\
\text { homologous semen on the } \\
\text { success rate of IUI }\end{array}$ & $\begin{array}{l}556 \text { couples with } 1401 \text { IUI } \\
\text { cycles }\end{array}$ & 16 features & Logistic regression & Small dataset \\
\hline $\begin{array}{l}\text { Assessing the effects of FSH } \\
\text { and clomiphene citrate } \\
\text { on infertile women with } \\
\text { unexplained infertility }\end{array}$ & \multicolumn{2}{|c|}{2259 IUI cycles of 684 couples 6 features } & Logistic regression & $\begin{array}{l}\text { A few determinative factors } \\
\text { studied }\end{array}$ \\
\hline Outcome prediction of ART & 257 infertile couples & 12 features & ANN & Small dataset \\
\hline $\begin{array}{l}\text { Prediction of implantation } \\
\text { after blastocyst transfer in } \\
\text { IVF or ICSI }\end{array}$ & 1052 patients in & 32 features & $\begin{array}{l}\text { Random Forest, Multivariate } \\
\text { logistic regression model }\end{array}$ & A small number of features \\
\hline
\end{tabular}

Most of the considered previous studies have used single classifiers and/or RF as a simple ensemble classifier. Some previous studies have illustrated that the stacked models can improve the classification performance for other applications and other datasets [19-21]. Therefore, in this study, a novel stacked ensemble is designed and proposed for improving the performance of IUI outcome prediction.

The main aim of this study is to develop an automatic classification and feature scoring method to predict intrauterine insemination (IUI) outcome and ranking of the most significant features, based on the features describing the couples and their corresponding IUI treatment cycles. For this purpose, a novel approach combining complex network-based feature engineering and stacked ensemble (CNFE-SE) is proposed. Three complex networks are extracted considering the patients' data similarities. The feature engineering step is performed on the complex networks. The original feature set and/or the features engineered are fed to the proposed stacked ensemble to classify and predict IUI outcome for couples per IUI treatment cycle. Our study is a retrospective study of a 5-year couples' data undergoing IUI. Data is collected from Reproductive Biomedicine Research Center, Royan Institute describing 11,255 IUI treatment cycles for 8,360 couples.

The main novelty of this study lies in three folds including:

- Proposing a method for feature scoring and classification based on weighted complex networks and stacking ensemble classifiers

- Proposing feature engineering method based on complex networks

- Designing a novel stacked ensemble classifier for predicting IUI outcome 
Table 2 List of the features engineered from the complex networks in this study

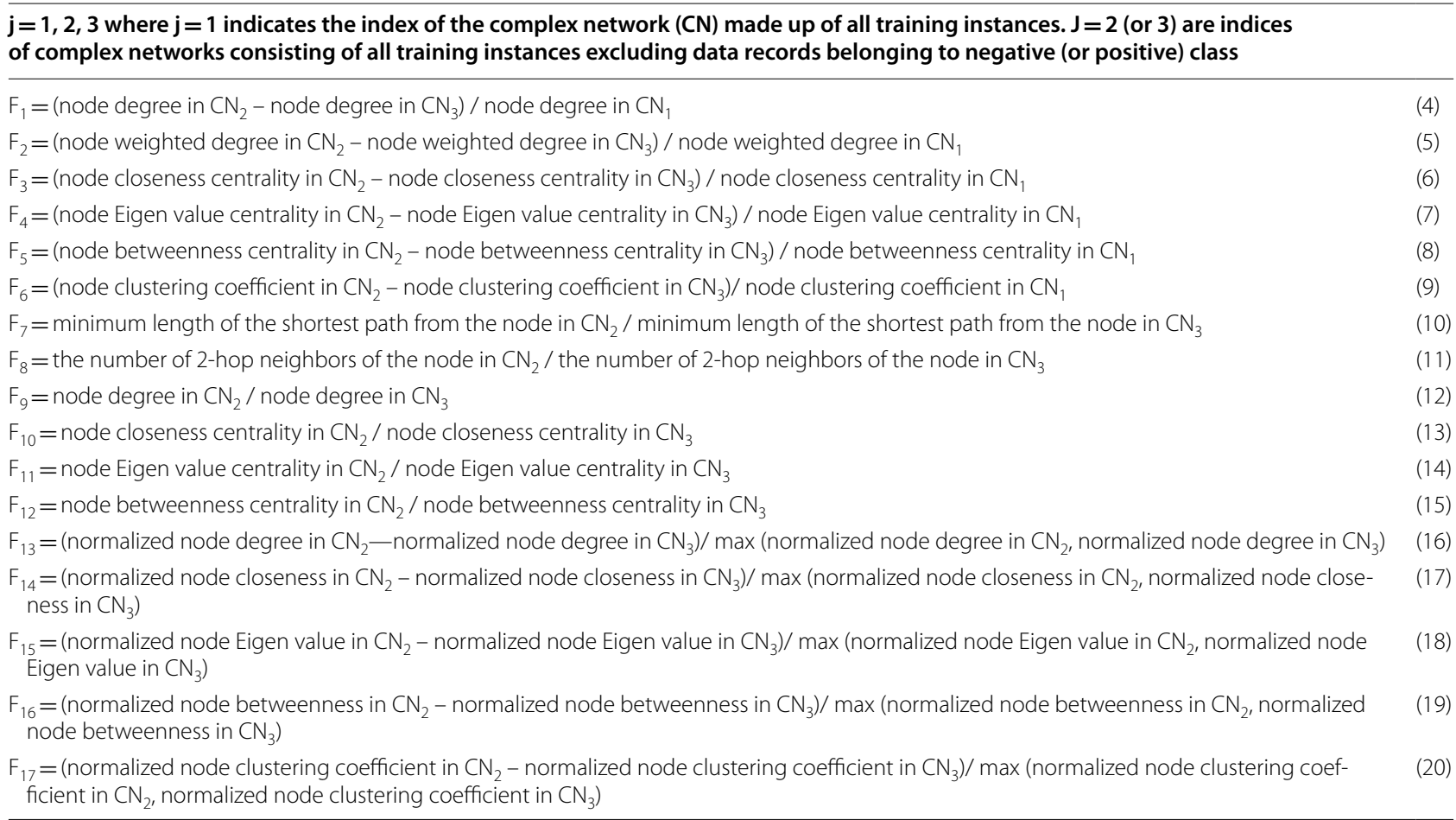

\section{Methods}

The main steps of the proposed approach combining complex network-based feature engineering and stacked ensemble (CNFE-SE) to predict the success of Intrauterine Insemination and ranking the features are illustrated in Fig. 1.

The main steps of the proposed method (CNFE-SE) as depicted in Fig. 1 include the modules for data collection and preparation, feature scoring and classification and finally model evaluation and validation. The first module consists of data collection, sampling from data, preprocessing the collected data and filtering irrelevant features. In the next module, ignoring a feature, constructing three complex networks from the patients, extracting features from the constructed complex networks, training the classifiers based on the extracted features and finally scoring the ignored feature are performed. The last module evaluates and validates the models trained in the previous module. More details about the mentioned tasks are described in the following subsections.

\section{Data collection}

Our research is approved by the Institutional Review Board of the Royan Institute Research Center and the Royan Ethics Committee consistent with Helsinki Declaration with the approval ID of IR.ACECR.ROYAN.REC.1398.213. Anonymity and confidentiality of data were respected.
Dataset studied in this article is collected from Royan Institute, a public none-profitable organization, affiliated to the academic center for education, culture and research (ACECR) in Iran. It includes the features describing the patients having been treated by IUI method in the Infertility clinic at Royan Institute between January 2011 and September 2015.

In this retrospective study, a completed episode is defined as a sequence of treatment cycles resulting in positive clinical pregnancy or when the treatment with IUI is stopped. The inclusion criteria for the couples to be treated under IUI cycles were male factor, ovulatory disorders such as PCOS, hypothalamic amenorrhea, diminished ovarian reserve, combined causes, and unexplained subfertility. The couples' duration of infertility was at least 1 year. Male infertility was defined as the semen quality parameters lower than the standards determined by WHO including sperm concentration lower than 15 million/ejaculate, semen volume lower than $1.5 \mathrm{~mL}$, and total motility lower than $40 \%$ [22]. The male partners with donor sperms, Varicocele, and semen samples with total motile sperm count lower than $1 \times 10^{6}$ were excluded from being candidates for IUI treatment. Additionally, patients with anatomical and metabolic abnormalities, severe endometriosis and/or systemic diseases were excluded from our study. 


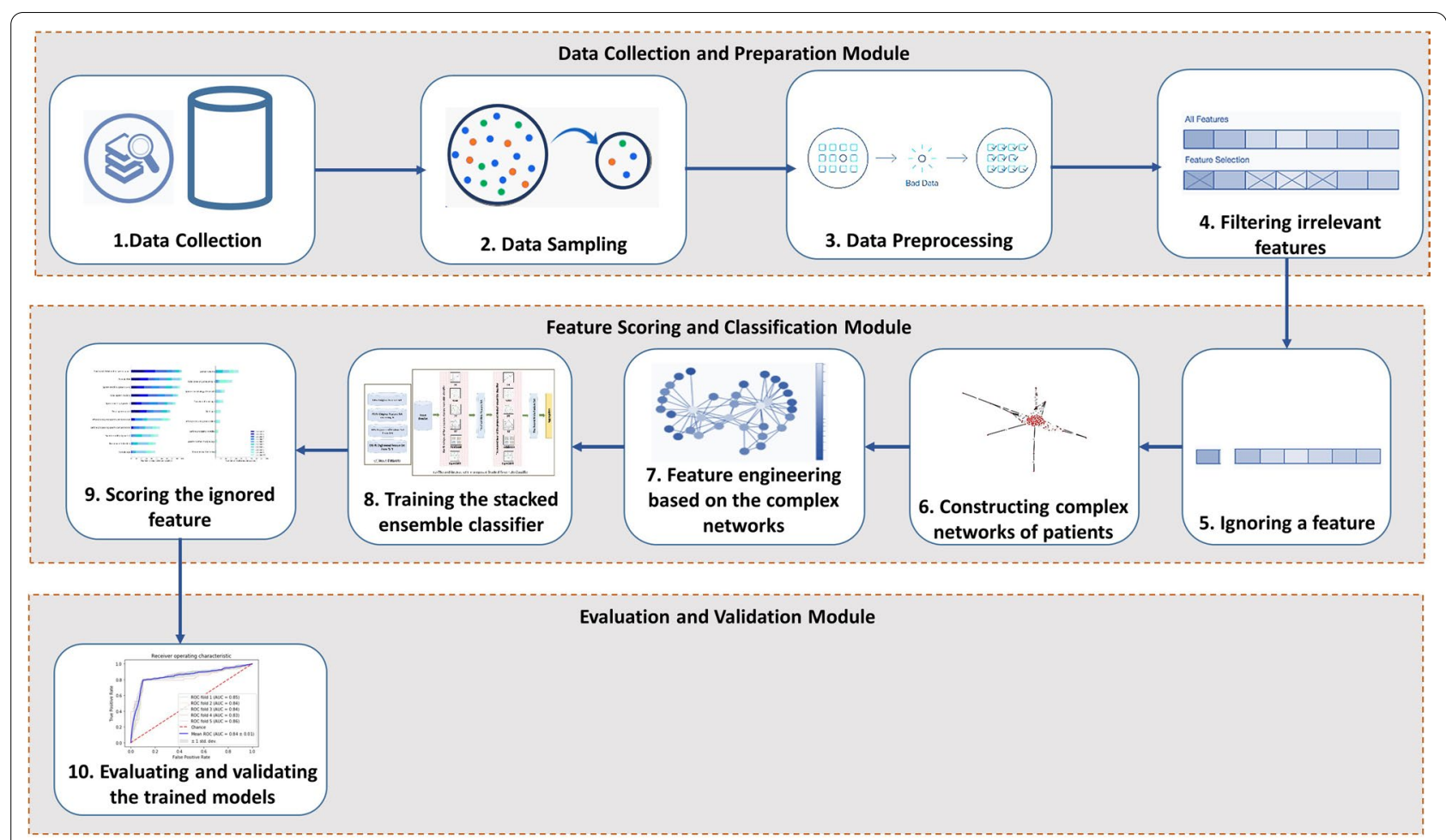

Fig. 1 The main steps of the proposed method (CNFE-SE) for feature scoring and classifying the patients to predict IUI outcome

11,255 IUI cycles related to 8,360 couples are considered in which the women age ranges from 16 to 47 with the average age of 29. This dataset contains 1,622 positive outcomes and 9,633 negative ones. Therefore, the overall pregnancy rate is $14.41 \%$ per completed cycle and $19.4 \%$ per couple. Each couple is treated for $1.31 \pm 0.59$ (mean \pm Standard Deviation) IUI cycles which ranges from 1 to 7 cycle.

The features describe the couples' demographic characteristics, historical data about their diseases, the clinical diagnosis, the treatment plans and the prescribed drugs to the couples, male semen quality, laboratory tests and the clinical pregnancy outcome. The considered demographic features include age, body mass index (BMI), education level, consanguinity with spouse and some other features. The information about the history of the patients' subfertility consists of the duration and type of infertility, length of marriage and so on.

The types of feature values are numerical, binary, nominal and binominal types for 86, 152, 51 and 7 features, respectively. More details about the features is shown in Appendix 1.

In the collected dataset, the majority of couples (almost $72 \%$ ) have been treated for one cycle, $22 \%$ of couples have underwent two cycles, $5 \%$ of couples have been treated for three cycles, and less than $1 \%$ have been treated more than three cycles. The maximum number of cycles for treating a couple is seven. Figure 2 depicts the distributions of positive and negative clinical pregnancy rates for patients per treatment cycle.

As illustrated by Fig. 2, 63\% of the couples belonging to the positive class (positive clinical pregnancy after completing the cycle) have been pregnant after the first treatment cycle. $26 \%$ of data records in the positive class have received positive outcome after the second cycle. Moreover, $74 \%$ of the couples in the negative class have been considered after the first cycle.

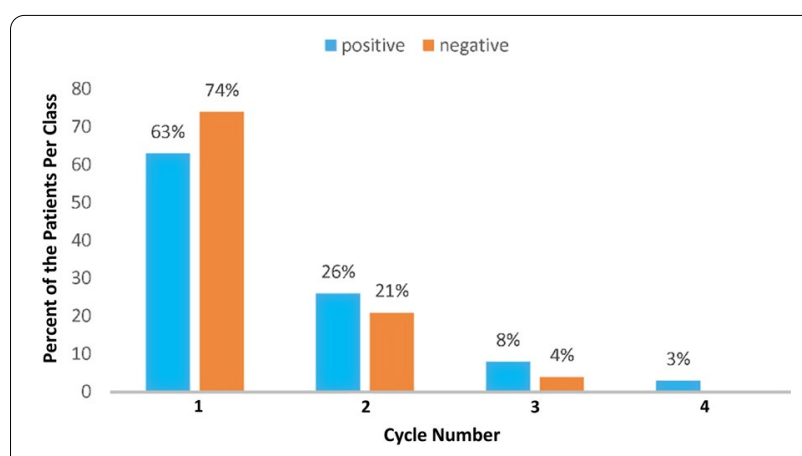

Fig. 2 The ratio of positive and negative clinical pregnancy per treatment cycle 


\section{Data sampling}

Data should be randomly partitioned into training and test datasets with no overlapping among these two subsets. The models are trained on the training dataset and finally are evaluated by applying them to the test datasets.

$\mathrm{K}$-fold cross validation (C.V.) is a common and popular sampling strategy used for this purpose. In this method, data is randomly divided into $\mathrm{K}$ disjoint equal-size subsets. Every time, one of these $\mathrm{K}$ subsets is considered as the test dataset and all (K-1) remaining subsets make the training one. The model is trained $\mathrm{K}$ times on $\mathrm{K}$ training datasets and applied to the corresponding test datasets to evaluate the performances of the trained models.

Before sampling from data, the features having missing value rate higher than $20 \%$ are removed from the study. Moreover, the patient records with high missing value rate (higher than 20\%) are excluded from the study and then, fivefold C.V. is used for sampling from the collected dataset, in this study.

At first, dataset is partitioned into non-overlapping subsets $D_{1}, D_{2}, \ldots, D_{K}$ based on K-fold Cross Validation strategy. Then, the models are trained on $\mathrm{K}$ training datasets composed of all $D_{1}, \ldots, D_{K}$ subsets excluding $D_{i}$ for $1 \leq \mathrm{i} \leq \mathrm{K}$. Therefore, the ith training dataset consists of all $D_{1}, \ldots, D_{K}$ but $D_{i}$ and the ith test dataset is $D_{i}$. The ith training dataset is balanced using over-sampling strategy.

Moreover, a strategy for classification structural risk assessment is used named as A-Test which will be described in the evaluation and validation subsection with more details. The number of instances of positive and negative outcomes in each folder of fivefold is 324325 and 1926-1927, respectively. therefore, the imbalance ratio of the training set in each of 5 -folds is about 0.168 .

\section{Data preprocessing}

Preprocessing of data is one of the most essential steps in the knowledge discovery tasks. A previous study have stated that $80 \%$ of total time in data mining projects is allocated for data preparation and preprocessing step [23].

In the first step, the initial collected dataset includes almost 86,000 data records describing the partners and about 1,000 features. The data records describing one couple per IUI treatment cycle are aggregated to make our dataset. Thus, the aggregated dataset includes 11,255 data records and 296 features describing a couple during an IUI treatment cycle.

The nominal features are converted to dummy binary variables. If a nominal features has $\mathrm{m}$ different levels or values, it will be converted to ( $m-1)$ dummy binary variables. Therefore, instead of considering a nominal feature in the classification and feature ranking, its corresponding dummy binary variables are considered in the mentioned tasks.

The missing values for numeric and categorical features are imputed based on the average and the most frequent values, respectively [24]. All numerical and ordinal features are normalized using min-max normalization method and the nominal features are converted into dummy binary variables.

Outlier detection is performed in this study based on isolation forest method which has been proposed by Liu et al. [25] as an appropriate outlier detection method for high dimensional data. The hyperparameters of Isolation Forest including the number of estimators, maximum number of the samples, contamination coefficient, maximum number of the features, bootstrapping or not, and the number of jobs are tuned using grid search method. For evaluating the performance of Isolation Forest, its results are compared to other outlier detection methods such as One-class SVM with kernel of Radial Basis Function (RBF), boxplot analysis and expert's opinions. Three outliers are identified by this method and excluded from the study.

\section{Filtering irrelevant features}

Since the aggregated dataset consists of many features, the irrelevant features can be removed to reduce the computational time required for processing and analyzing data. Thus, the features having very low correlation with the output feature or very high correlation with other input features are excluded from this study. The linear correlation coefficient between pairs of the features $\mathrm{F}_{\mathrm{p}}$ and $\mathrm{F}_{\mathrm{q}}$ are calculated as Eq. (1):

$$
\operatorname{Corr}\left(F_{p} F_{q}\right)=\sum_{i} \frac{\left(F_{i p}-m_{p}\right)\left(F_{i q}-m_{q}\right)}{\sqrt{\sum_{j}\left(F_{j p}-m_{p}\right)^{2}} \sqrt{\sum_{j}\left(F_{j q}-m_{q}\right)^{2}}}
$$

where $F_{x, p}\left(F_{x, q}\right)$ indicates the xth row of the feature $F_{p}$ $\left(\mathrm{F}_{\mathrm{q}}\right)$ and $\mathrm{m}_{\mathrm{p}}\left(\mathrm{m}_{\mathrm{q}}\right)$ denotes the average of the feature $\mathrm{F}_{\mathrm{p}}$ $\left(\mathrm{F}_{\mathrm{q}}\right)$, respectively.

If two features $\mathrm{F}_{\mathrm{p}}$ and $\mathrm{F}_{\mathrm{q}}$ have low (high) correlation, Corr $\left(F_{p}, F_{q}\right)$ tends to zero $(-1$ or +1$)$.

\section{Ignoring a feature}

Breiman has proposed measuring the feature importance by mean decrease in accuracy (MDA) of random forest [26]. This study aims at ranking the features according to their predictive power for classifying the instances to positive or negative clinical pregnancy. For this purpose, all the steps 6-9 are performed by considering all the features excluding one feature each time and MDA for the trained proposed classifier is calculated on the validation dataset. MDA values show the amount of reducing the model accuracy after removing a feature. Therefore, the higher values 
of MDA indicate the higher predictive ability of the corresponding features.

\section{Constructing complex networks of patients}

For modeling nonlinear data, complex networks are effective method [27]. Complex network is a weighted undirected graph $\mathrm{G}=(\mathrm{V}, \mathrm{E}, \mathrm{W})$, where $\mathrm{V}$ is the set of nodes, $\mathrm{E}$ denotes the set of edges e $\left(v_{i}, v_{j}\right)$ between the pairs of the nodes $\mathrm{v}_{\mathrm{i}}$ and $\mathrm{v}_{\mathrm{j}}$ and $\mathrm{W}$ is the weights $\mathrm{w}\left(\mathrm{v}_{\mathrm{i}}, \mathrm{v}_{\mathrm{j}}\right)$ assigned to their corresponding edges $\mathrm{e}\left(\mathrm{v}_{\mathrm{i}}, \mathrm{v}_{\mathrm{j}}\right)$ of $\mathrm{E}$.

Three complex networks are constructed from the training datasets and one data record which should be classified independent from it belongs to training or test dataset. The first one is comprised of all the training data records and one data record which should be classified as its nodes and is called CN1. The second and the third complex networks consist of one data record which should be classified and
Feature engineering based on the complex networks In this section, three complex networks per data record are constructed including the considered data record, all training instances as $\mathrm{CN} 1$ and all training instances excluding negative (positive) instances as CN2 (CN3). A simple intuitive hypothesis is that a node has more similarity with the training instances of its own class compared to the instances of the other class. Therefore, the node centrality in different complex networks CN1, CN2 and $\mathrm{CN} 3$ can be compared to classify the node. Features listed in Tables 3, 4 are defined based on this hypothesis.

Node degree is the number of its adjacent edges. Betweenness centrality for graph nodes have been introduced by Bavelas [28] and is calculated as Eq. (4). If a node lies in many shortest paths between pairs of nodes, its Betweenness centrality will be high. Nodes with high Betweenness centrality are the bridges for information flow.

$$
\text { Betweenness }\left(v_{i}\right)=\sum_{j<k} \frac{\text { number of the shortest paths between } v_{j} \text { and } v_{k} \text { passing } v_{i}}{\text { number of the shortest paths between } v_{j} \text { and } v_{k}}
$$

all training data records excluding the negative and positive classes and named as $\mathrm{CN} 2$ and $\mathrm{CN} 3$, respectively. If the considered data record belongs to training dataset, its class label is excluded from its corresponding complex networks.

In other words, the nodes of CN1, CN2 and CN3 are one data record which should be classified and all the training data records, positive labeled and negative labeled training data records, respectively. Therefore, for each data record, three complex networks are constructed.

An edge between node $v_{i}$ and $v_{j}$ is drawn if the distance between the input features of the ith and jth training data records is smaller than a user-defined threshold. For calculating the pairwise distance between data records, Euclidean distance function is used and can be calculated as Eq. (2):

$$
\operatorname{Distance}\left(v_{i} v_{j}\right)=\sqrt{\sum_{p=1}^{m}\left(F_{i p}-F_{j p}\right)^{2}}
$$

where $m$ is the number of the input features, $F_{i, p}$ and $F_{i, p}$ denote the pth input feature values for data records corresponding to $v_{i}$ and $v_{j}$.

The weight of the edge $\mathrm{e}\left(\mathrm{v}_{\mathrm{i}}, \mathrm{v}_{\mathrm{j}}\right)$ is calculated as Eq. (3):

$$
w\left(v_{i} v_{j}\right)=\frac{\operatorname{distance}\left(v_{i} v_{j}\right)}{\max \left(\operatorname{distance}\left(v_{k} v_{h}\right) ; v_{k} v_{h} \in V\right)}
$$

Node closeness centrality measures the reciprocal of the sum of the length of the shortest paths between the node and all other nodes in the graph.

\section{Table 3 MDA values of top-20 features}

\begin{tabular}{ll}
\hline Feature & $\begin{array}{l}\text { Mean decrease } \\
\text { in accuracy } \\
\text { (MDA) }\end{array}$ \\
\hline Post wash total motile sperm count & 5.8 \\
Female BMI & 5.2 \\
Sperm motility (grade $\mathrm{a}+\mathrm{b}$ ) & 5 \\
Total sperm motility & 4.9 \\
Sperm motility (grade c) & 4.7 \\
Total sperm count & 4.5 \\
After processing sperm concentration & 4.3 \\
Before processing sperm concentration & 3.9 \\
Sperm motility (grade d) & 3.7 \\
Male age & 3.6 \\
Semen volume & 3.4 \\
Duration of infertility & 3.3 \\
Total dose of gonadotropin & 2.9 \\
Female age & 2.7 \\
Duration of marriage & 2.5 \\
Sperm morphology (Amorph) & 2.5 \\
After processing progression & 2.4 \\
Before processing motility & 2.3 \\
Sperm normal morphology & 2.2 \\
Endometrial thickness & 2.1 \\
\hline
\end{tabular}


Table 4 Comparing the performance of CNFE-SE with other state of the art classifiers

\begin{tabular}{lllllll}
\hline Feature set & Classifier & Accuracy & Sensitivity & Specificity & AUC & F Score \\
\hline All 296 features & RF & $0.58 \pm 0.01$ & $0.69 \pm 0.05$ & $0.46 \pm 0.06$ & $0.58 \pm 0.01$ & $0.55 \pm 0.05$ \\
& DT & $0.55 \pm 0.01$ & $0.62 \pm 0.04$ & $0.49 \pm 0.04$ & $0.55 \pm 0.01$ & $0.55 \pm 0.04$ \\
& NB & $0.53 \pm 0.01$ & $0.79 \pm 0.11$ & $0.26 \pm 0.12$ & $0.54 \pm 0.01$ & $0.39 \pm 0.11$ \\
& ANN & $0.50 \pm 0.01$ & $0.54 \pm 0.16$ & $0.45 \pm 0.16$ & $0.50 \pm 0.01$ & $0.49 \pm 0.16$ \\
& SVM & $0.54 \pm 0.01$ & $0.28 \pm 0.1$ & $0.8 \pm 0.09$ & $0.56 \pm 0.01$ & $0.41 \pm 0.05$ \\
& XGboost & $0.55 \pm 0.01$ & $0.53 \pm 0.03$ & $0.56 \pm 0.03$ & $0.55 \pm 0.01$ & $0.54 \pm 0.03$ \\
& LGBM & $0.60 \pm 0.01$ & $0.59 \pm 0.03$ & $0.59 \pm 0.01$ & $0.64 \pm 0.01$ & $0.59 \pm 0.02$ \\
& Adaboost & $0.59 \pm 0.01$ & $0.69 \pm 0.02$ & $0.48 \pm 0.02$ & $0.60 \pm 0.01$ & $0.56 \pm 0.02$ \\
Cnly most important & $0.71 \pm 0.01$ & $0.69 \pm 0.01$ & $0.73 \pm 0.01$ & $0.71 \pm 0.01$ & $0.71 \pm 0.01$ \\
features & CNFE-SE without FE & $0.85 \pm 0.01$ & $0.79 \pm 0.01$ & $0.91 \pm 0.01$ & $0.84 \pm 0.01$ & $0.85 \pm 0.01$ \\
& RF & $0.60 \pm 0.02$ & $0.69 \pm 0.03$ & $0.50 \pm 0.02$ & $0.59 \pm 0.02$ & $0.60 \pm 0.02$ \\
& DT & & & $0.037 \pm 0.02$ & $0.58 \pm 0.03$ \\
& NB & $0.57 \pm 0.03$ & $0.63 \pm 0.01$ & $0.54 \pm 0.04$ & 0.57 \\
& ANN & $0.54 \pm 0.01$ & $0.52 \pm 0.01$ & $0.57 \pm 0.01$ & $0.54 \pm 0.01$ & $0.54 \pm 0.01$ \\
& SVM & $0.54 \pm 0.01$ & $0.55 \pm 0.01$ & $0.52 \pm 0.01$ & $0.53 \pm 0.01$ & $0.53 \pm 0.01$ \\
XGboost & $0.58 \pm 0.01$ & $0.51 \pm 0.01$ & $0.70 \pm 0.01$ & $0.60 \pm 0.01$ & $0.61 \pm 0.01$ \\
& LGBM & $0.58 \pm 0.01$ & $0.57 \pm 0.01$ & $0.59 \pm 0.01$ & $0.58 \pm 0.02$ & $0.58 \pm 0.01$ \\
& Adaboost & $0.62 \pm 0.02$ & $0.61 \pm 0.02$ & $0.63 \pm 0.03$ & $0.62 \pm 0.02$ & $0.62 \pm 0.02$ \\
CNFE-SE without FE & $0.62 \pm 0.01$ & $0.69 \pm 0.01$ & $0.51 \pm 0.01$ & $0.61 \pm 0.01$ & $0.60 \pm 0.01$ \\
CNFE-SE with FE & $0.72 \pm 0.01$ & $0.71 \pm 0.01$ & $0.74 \pm 0.01$ & $0.72 \pm 0.01$ & $0.72 \pm 0.01$ \\
& & $0.82 \pm 0.01$ & $0.92 \pm 0.01$ & $0.87 \pm 0.01$ & $0.87 \pm 0.01$ \\
\hline
\end{tabular}

Node Eigen vector centrality is higher when the node is pointed to by many important nodes.

Clustering coefficient of a node is calculated as Eq. (5):

$$
\begin{aligned}
& \text { Clustering Coefficient }\left(v_{i}\right) \\
& =\frac{\text { number of triangles connected to } v_{i}}{\text { number of triples centered around } v_{i}}
\end{aligned}
$$

Since, the number of the instances are very high, the complex networks are partitioned into smaller communities to reduce the computational complexity for calculating the engineered features.

One complex network extracted from only 100 data records treated by IUI method as a sample is shown in Fig. 3.

Figure 4 depicts two complex networks of the same samples of positive instances drawn by different thresholds.

As shown by Fig. 4, reducing the threshold for keeping the edges in the complex network even with a small value lead to the network with more sparsity and more smallsized communities.

Figure 5 illustrates three complex networks from the samples of both classes, negative and/or positive classes.

As shown by Fig. 5, for the same thresholds, complex network considering the instances of both classes has the most density and the complex network from only positive instances has the most sparsity and consists of several small communities.

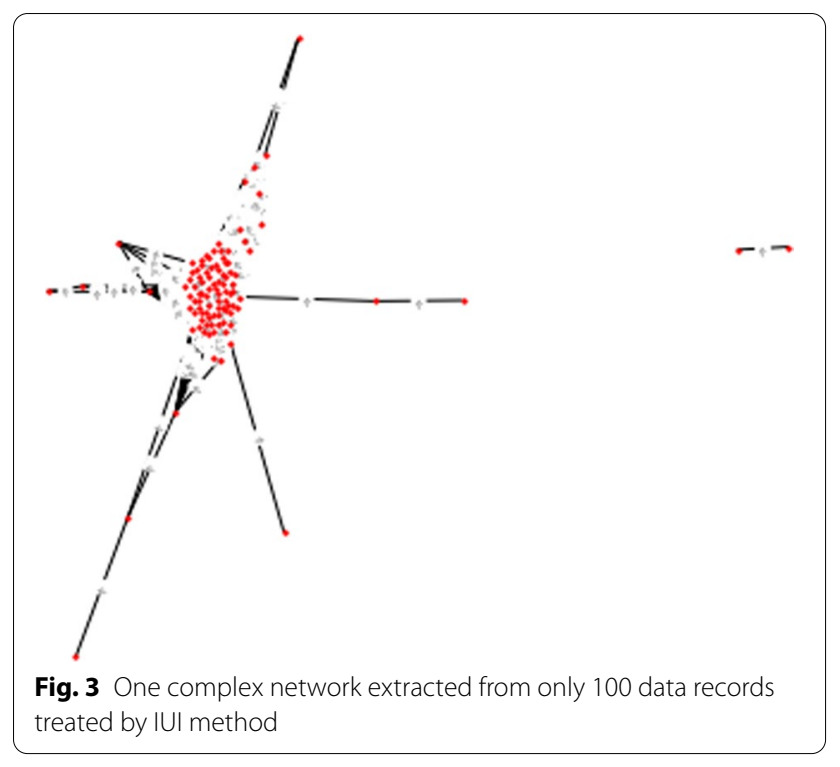

\section{Training the stacked ensemble classifier}

Stacked ensemble classifier which is a scalable meta-modeling methodology has been first introduced by Wolpert in 1994 [29]. It has been inspired by neural networks whose classifiers have been considered as the nodes. Instead of a linear model, the stacked classifier can use any base classifier. The stacking operation has been performed by either a normal stacking or a re-stacking mode. 


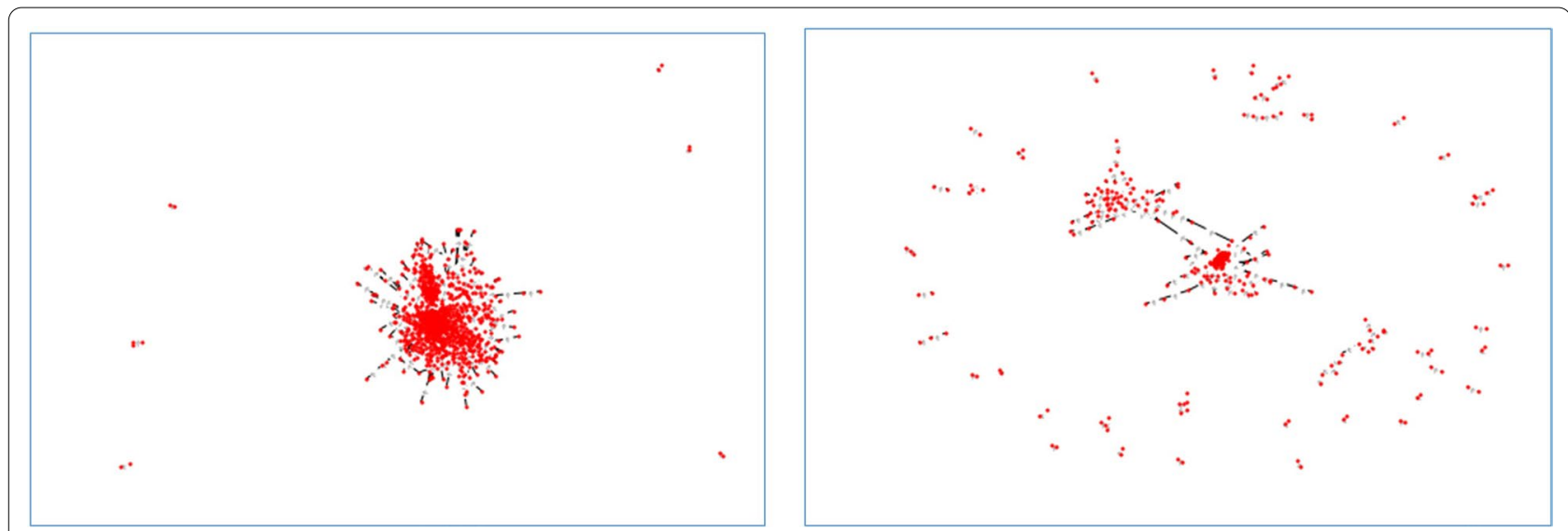

Fig. 4 Two complex networks drawn from the positive training data samples by a threshold of $0.7^{*}$ average of the distance Matrix, $\mathbf{b}$ threshold of $0.5 *$ average of the distance matrix

In the normal stacking mode, the base classifiers in each layer use the output scores of the previous ones as the predictors similar to a typical feedforward neural network. The formula of normal stacking mode is written as Eq. (6):

$$
\begin{aligned}
f_{n}(x V)= & V_{n k}\left(f_{n-1}\left(x V_{n-11}\right) f_{n-1}\left(x V_{n-12}\right), \ldots,\right. \\
& \left.f_{n-1}\left(x V_{n-1 D_{n-1}}\right)\right)
\end{aligned}
$$

where $\mathrm{n}$ indicates the $\mathrm{nth}$ layer of the stacked ensemble, $\mathrm{x}$ denotes a sample of a dataset, $\mathrm{V}$ presents a vector holding the neurons (the base classifiers), D is the number of hidden neurons through the nth hidden layer and finally, $\mathrm{k}$ is the kth neuron in the nth layer.

Some previous studies have illustrated that the stacked models can improve the performance of the classification $[20,21,30]$. Therefore, in this study, a new stacked ensemble classifier is proposed and designed based on the normal stacking mode. In the beginning, some of the basic classifiers are trained, and those outperforming the others are selected to be considered as the base classifiers in the stacked ensemble layers. The architecture of the proposed stacked ensemble classifier is shown in Fig. 6.

As illustrated in Fig. 6, input dataset consists of the features in OFS, FS-Fi, EFS and/or EFS-Fi. Input dataset is fed to the base classifiers in the first layer of the proposed stacked ensemble classifier.

Several different classifiers are trained and verified. The classifiers for using in the ensemble layers of our proposed stacked ensemble classifier are chosen among different trained classifiers with different values of hyperparameters based on their accuracy and diversity on the validation dataset. A previous study has proposed a method to choose classifiers for ensemble learning based on accuracy and diversity which is used in this study for the same purpose. The pairwise diversity of the classifiers are calculated using $Q$ statistic.
Logistic regression (LR) [31], support vector machines (SVM) [32], decision tree (DT) [33], random forest (RF) [26], Adaboost [34] and LightGradient Boosting Machine (LightGBM) [35] are the base classifiers chosen based on their accuracy and diversity in both ensemble layers.

LR, SVM with linear kernel and DT are appropriate classifiers for classifying linearly separable data. SVM with non-linear kernels, RF, Adaboost and LightGBM are ensemble classifiers which can classify nonlinearly separable data with high performance. All the mentioned classifiers can be trained fast. Therefore, they are chosen as the base classifiers of the proposed stacked ensemble classifier.

The hyperparameters of the classifiers are tuned based on grid search method and the best values for hyperparameters leading to the highest accuracy for validation dataset are considered for each classifier.

After training the base classifiers in the first layer, their outputs are considered as Meta features according to the normal stacking mode. The Meta features are fed into the base classifiers of the second layer for training them. Finally, the outputs of the base classifiers in the second layer are aggregated by weighted voting aggregation rule.

The weight of each base classifier is obtained by measuring its accuracy for classifying the validation dataset. The validation dataset is about $20 \%$ of the original training dataset which is excluded during the base classifiers' training in both layers.

Mathematical calculation is performed in this study to show the performance improvement obtained by stacked ensemble compared to traditional one-layer ensemble and the individual classifiers.

Without loss of generality, it is assumed that each base classifier in the first layer of stacked ensemble has the error rate of $\varepsilon<0.50$. If the aggregation of the base classifiers is performed with bagging strategy which is the 

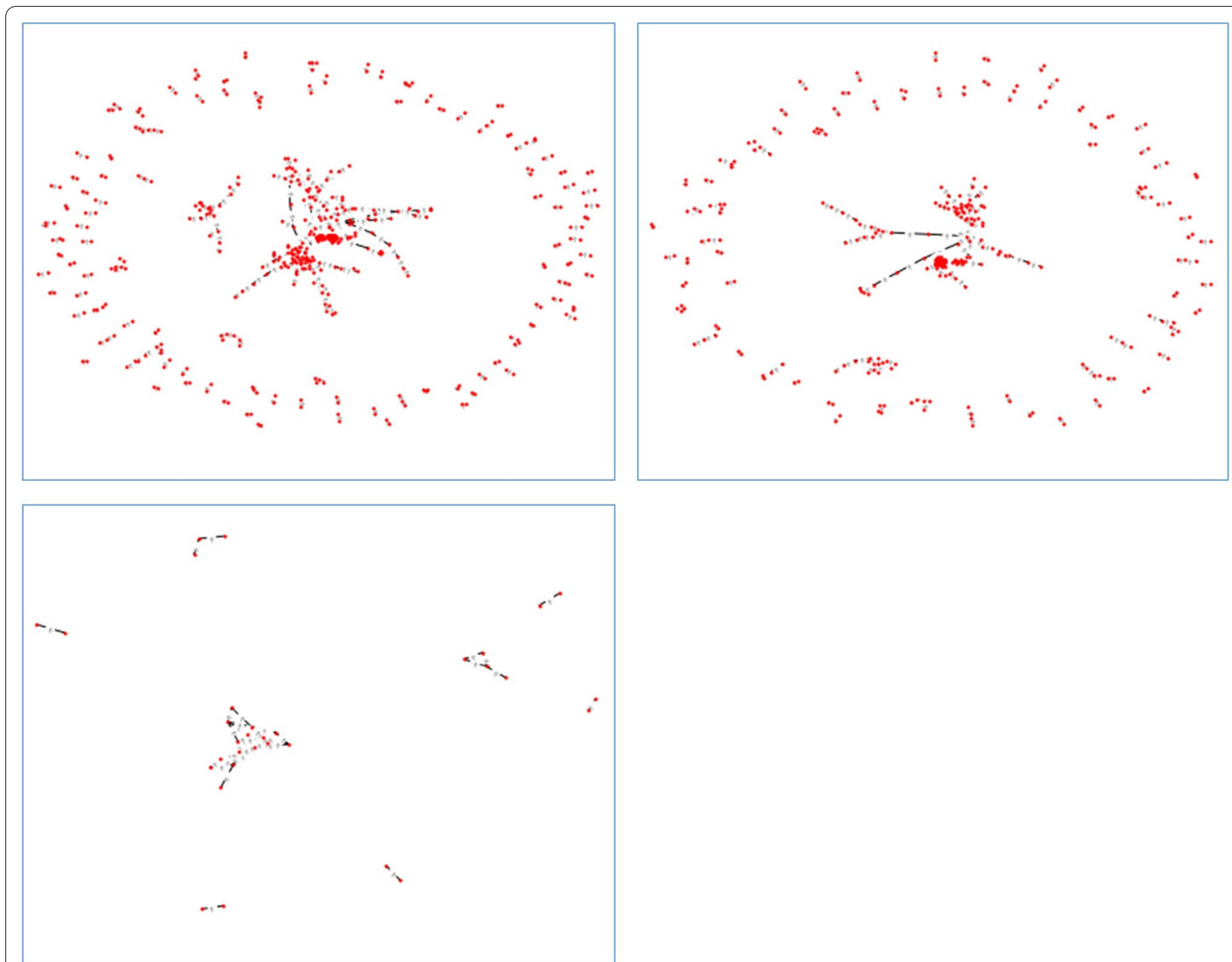

Fig. 5 Three complex networks extracted from the samples of $\mathbf{a}$ both classes, $\mathbf{b}$ negative class, and $\mathbf{c}$ positive class with the same threshold

simplest aggregation method and uses majority voting, the error rate of the first ensemble layer $\left(\varepsilon_{\mathrm{L} 1}\right)$ can be calculated as Eq. (7):

$$
\varepsilon_{L 1}=\sum_{i=\left(\frac{M}{2}+1\right)}^{M}\left(\begin{array}{c}
M \\
i
\end{array}\right)^{i} \varepsilon^{i}(1-\varepsilon)^{M-i}
$$

where $M$ is the number of the base independent classifiers in the first ensemble layer. For misclassifying a data record using bagging strategy as the aggregation method, more than half of the base classifiers should misclassify the record. If it is assumed that $i$ is the number of the base classifiers which misclassify the data record, i should be more than $M / 2$ for misclassifying it with the first ensemble layer. For example, if $M$ is 25 , at least 13 base classifiers should misclassify data for erroneous classifying data in ensemble of these base classifiers. Now, if $\varepsilon$ is 0.35 for each of 25 base classifiers, $\varepsilon_{\mathrm{L} 1}$ will be 0.04 . It shows the first layer of ensemble or traditional ensemble can improve the error rate of the single independent classifiers significantly.

Now, it is assumed that we have one more ensemble layer such as a two-layer stacked ensemble. Bagging strategy uses simple majority voting for classifying data as Eq. (8):

$$
\text { classLabel }_{\text {ensemble }}\left(r_{j}\right)=\left\{\begin{array}{l}
\text { Positive if } \sum_{i=1}^{M} \delta\left(\text { classLabel }_{i}\left(r_{j}\right)==\text { Positive }\right)>\frac{M}{2} \\
\text { Negative otherwise }
\end{array}\right\}
$$




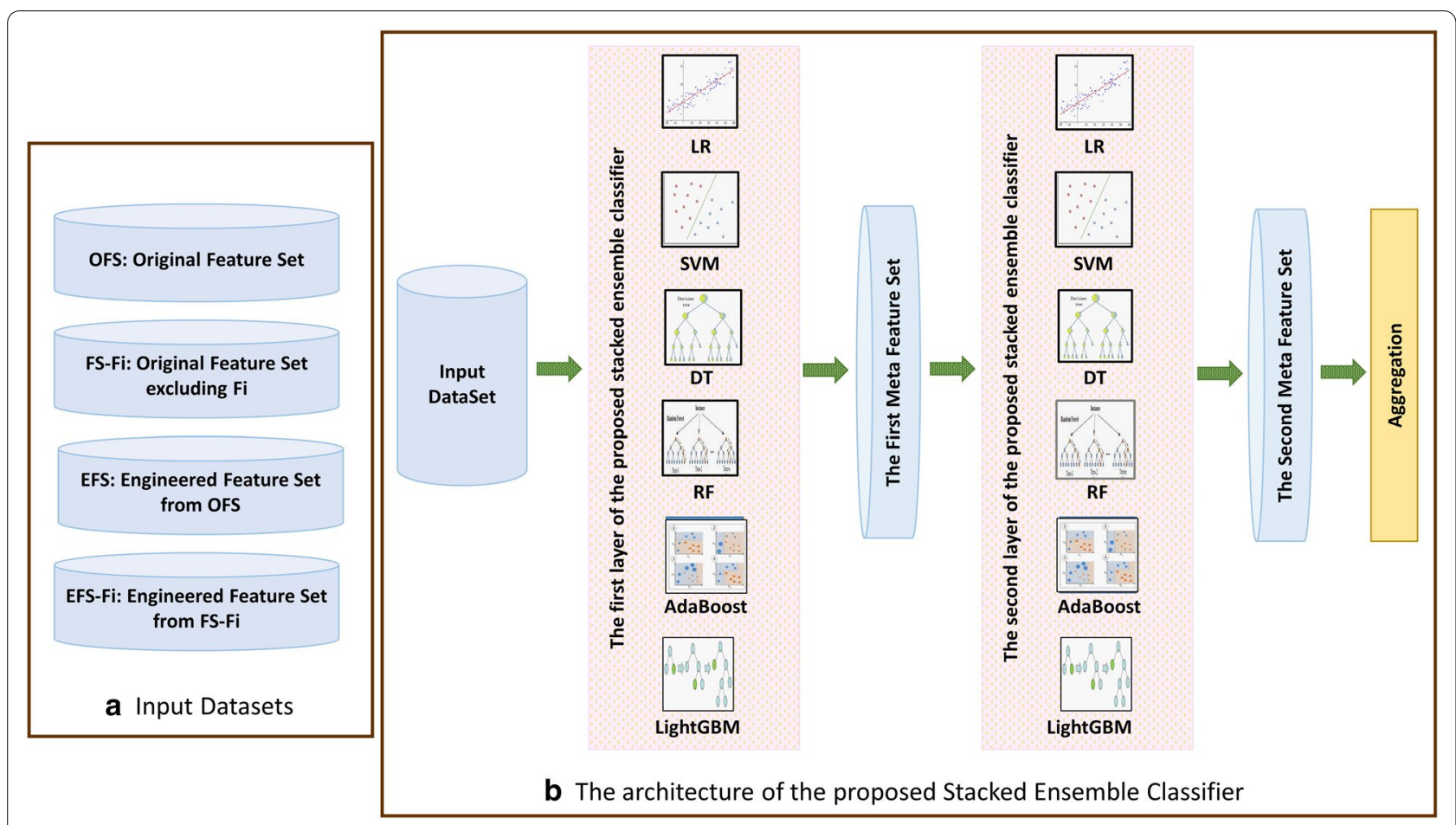

Fig. $\mathbf{6}$ a Input datasets, and $\mathbf{b}$ the architecture of the proposed Stacked Ensemble classifier

where $r_{j}$ indicates the $j$ th data record and $i$ denotes the ith base classifier. As shown in Eq. (8), a simple decision tree or SVM with linear kernel can provide rules or find hyperplanes to classify data according to Eq. (8). Therefore, it can be shown that the performance of each base classifier in the second layer will not be worse than the simple bagging aggregation strategy used in the first ensemble layer.

This conclusion is true because each base classifier will try to find the hyperplane or rules to discriminate the training samples of two classes. But, bagging strategy uses simple majority voting. Furthermore, the input features (the first meta feature set as shown by Fig. 6) for the base classifiers of the second ensemble layer are the same as the input features fed to the bagging strategy in the first ensemble layer. These input features are the output class labels generated by the base classifiers in the first layer. Therefore, the error rate of each base classifier in the second ensemble layer would be at most $\varepsilon_{\mathrm{L} 1}$.

The aggregation rule in the first ensemble layer is majority voting in the bagging strategy. The base classifiers try to separate the instances of different classes using linear or non-linear hyperplanes or rules. The input dataset for majority voting in the first ensemble layer is the first meta feature set. Therefore, the input of the majority voting rule and the base classifiers of the second ensemble layer is the same. The majority voting rule can be stated as Eq. (9) for the first meta feature set with $M$ columns:

$$
\text { label }_{M V}\left(r_{j}\right)=\left\{\begin{array}{l}
\text { Positive if } \sum_{i=1}^{M} \text { classLabel }_{i}\left(r_{j}\right)>0 \\
\text { Negative }
\end{array}\right\}
$$

where $\mathrm{MV}$ is the majority voting strategy. Majority voting strategy is similar to using a hyperplane considering the equal coefficients for all of its input features as the separator of two classes.

The base classifiers try to find a best hyperplane for discriminating the instances of two classes. Therefore, their fitted hyperplane will not be worse than the hyperplane used with majority voting strategy. Thus, their performance will be more than or equal to the performance of the majority voting in the first ensemble layer. According to the Eq. (7), it is shown that the performance of the majority voting will be much better than the performance of the single classifiers in the first ensemble layer. Therefore, the performance of the single classifiers in the second ensemble layer will be better than the performance of the single classifiers in the first ensemble layer.

According to Eq. (7), if the bagging strategy is used for the second ensemble layer, the error rate of the second ensemble layer in the stacked ensemble would be $\varepsilon_{\mathrm{L} 2}$ which can be calculated as Eq. (9): 


$$
\varepsilon_{L 2}=\sum_{j=\left(\frac{M 2}{2}+1\right)}^{M 2}\left(\begin{array}{c}
M 2 \\
j
\end{array}\right) \varepsilon_{b 2}^{j}\left(1-\varepsilon_{b 2}\right)^{M 2-j} \leq \sum_{j=\left(\frac{M 2}{2}+1\right)}^{M 2}\left(\begin{array}{c}
M 2 \\
j
\end{array}\right) \varepsilon_{L 1}^{j}\left(1-\varepsilon_{L 1}\right)^{M 2-j}
$$

where $M_{2}$ is the number of the base classifier in the second ensemble layer of the stacked ensemble and $\varepsilon_{\mathrm{b} 2}$ is the error rates of the base classifiers in the second ensemble layer. As mentioned in the previous paragraph, the error rate of each base classifier in the second layer would be at most $\varepsilon_{\mathrm{L} 1}$. Therefore, $\varepsilon_{\mathrm{b} 2}$ will be not more than $\varepsilon_{\mathrm{L} 1}$.

According to Eq. (7) and Eq. (9), the relationship among $\varepsilon, \varepsilon_{\mathrm{L} 1}$ and $\varepsilon_{\mathrm{L} 2}$ can be shown in Eq. (10):

$$
\varepsilon_{L 2} \ll \varepsilon_{L 1} \ll \varepsilon
$$

A previous study have demonstrated that adding more layers to stack ensemble can improve the classification performance in terms of accuracy and AUC [1].

Based on the obtained results, it can be shown that adding more layers to stacked ensemble can improve its performance. Although, adding more layers has higher burden of time complexity and memory usage, too.

There are a few studies considering the effect of the ensemble size or cardinality (the number of the base classifiers in the ensemble classifier) on the performance of the ensemble method $[1,2]$. The previous studies have shown the ensemble size depends on the diversity of the base classifiers included in the ensemble and its aggregation rule $[1,2]$. In addition, a previous study has examined different ensemble sizes including 10, 20, 50 and 100 classifiers for bioinformatics applications [3]. They have shown that the best ensemble size has been 50 but the ensemble size of 10 is sufficient to achieve to highly reasonable performance [3]. used in this study including Accuracy, Sensitivity, Specificity and F-Score as shown in Eq. (11) -(14):

$$
\begin{aligned}
& \text { Accuracy }=\frac{T P+T N}{N} \\
& \text { Sensitivity }=\frac{T P}{T P+F N} \\
& \text { Specificity }=\frac{T N}{T N+F P} \\
& F-\text { Score }=2 \times \frac{\text { Sensitivity } \times \text { Specificity }}{\text { Sensitivity }+ \text { Specificity }}
\end{aligned}
$$

where TP and FP (TN and FN) indicate the number of instances in the positive (negative) classes which are classified correctly and incorrectly, respectively.

Moreover, the area under the curve (AUC) of the receiver operating curve (ROC) is considered.

In order to validate the results, the experiments are repeated 50 times, and each time the data is selected based on fivefold C.V.

A novel method named as A-Test has been proposed in a previous study to calculate the structural risk of a classifier model as its instability with the new test data [36]. A-test calculates the misclassification error percentage $\Gamma_{\zeta, \mathrm{K}}$ for different $\mathrm{K}$ values using the balanced $\mathrm{K}$-fold validation. In this study, the values of $\Gamma_{\zeta, \mathrm{K}}$ will be reported for different classifiers and different feature sets. $\Gamma_{\zeta, \mathrm{K}}$ is calculated as Eq. (15):

$$
\Gamma_{\zeta, \mathrm{K}}=\frac{100}{N}\left(\begin{array}{c}
\sum_{i=1}^{N} \delta((\text { predictedLabel }=\text { Negative }) .(\text { realLabel }=\text { Positive })) \\
+\sum_{i=1}^{N} \delta((\text { predictedLabel }=\text { Positive }) .(\text { realLabel }==\text { Negative }))
\end{array}\right)
$$

\section{Scoring the ignored feature}

As mentioned in Sect. 1.5, MDA score is calculated for each feature and is considered as the feature importance score.

\section{Evaluating and validating the trained models}

To evaluate the performances of the trained models, the performance measures for classification problems are

$$
K=2 \ldots K_{\max }
$$

where $\mathrm{K}_{\max }$ cannot be more than the size of the minority class. For estimating the structural risk of a classifier method, the average of the values of $\Gamma_{\zeta, \mathrm{K}}$ is considered as Eq. (16):

$$
\Gamma_{\zeta}^{\wedge}=\frac{\sum_{K=2}^{K_{\max }} \Gamma_{\zeta \cdot K}}{K_{\max }-1}
$$


where $\Gamma_{\zeta}^{\wedge}$ ranges from 0 to $100 \%$ which higher values show higher risk of classification and lower values show the higher capacity and generalization ability of the model. Therefore, the lower values of $\Gamma_{\zeta} \wedge$ are more desired.

\section{Experimental results}

In this section, the features are ranked based on MDA obtained by ignoring them during the training of CNFE$\mathrm{SE}$. Then the partial dependencies between high-ranked features are discussed. Finally, the performance of the proposed model (CNFE-SE) is compared with other state-of-the-art classifiers.

\section{Ranking the significance of features}

Figure 7 represents top- 20 important features with highest MDA score for IUI outcome prediction based on 50 repetitions of CNFE-SE training on different training samples. Post wash total motile sperm counts, female BMI, sperm motility grades $a+b$, total sperm motility and sperm motility grade $\mathrm{c}$ are high-ranked predictors of IUI outcome. Additionally, post-wash total motile sperm counts, female BMI, and total sperm counts are the features illustrated with dark blue colors in Fig. 7, have the highest repetitions as the first informative features. Generally, the variables related to the men's semen analysis parameters are high-ranked features in this study.
The Pearson correlation coefficients are calculated among the top-20 important features, and Fig. 8 depicts the heat map of the correlation coefficients.

As shown by Fig. 8, the male semen parameters are positively correlated to each other, the more sperm concentration, the more total sperm count, and the more total motile sperm count. Also, couples' duration of infertility and duration of marriage are positively correlated.

Figure 9 shows the exact values of MDA score for top20 features in this study.

In addition, Table 3 lists MDA values of top-20 features.

\section{Partial dependency between the features}

Figure 10 depicts the partial dependency plots for the most important features. Partial dependency plots show whether a feature has a positive or negative effect on the response variable when the other ones are controlled. However, in order to interpret the graphs, we should note that changes in the clinical pregnancy probabilities in terms of the value of the features, even the most significant ones, are roughly small (the $y$-axis range is $0.44-0.52$ ). Therefore, it is noteworthy that none of the features could individually and significantly alter the pregnancy rates more than 0.52 . This finding underlines the value of the machine learning approach by determining the complicated association between individual predictors to make an effective classification model.
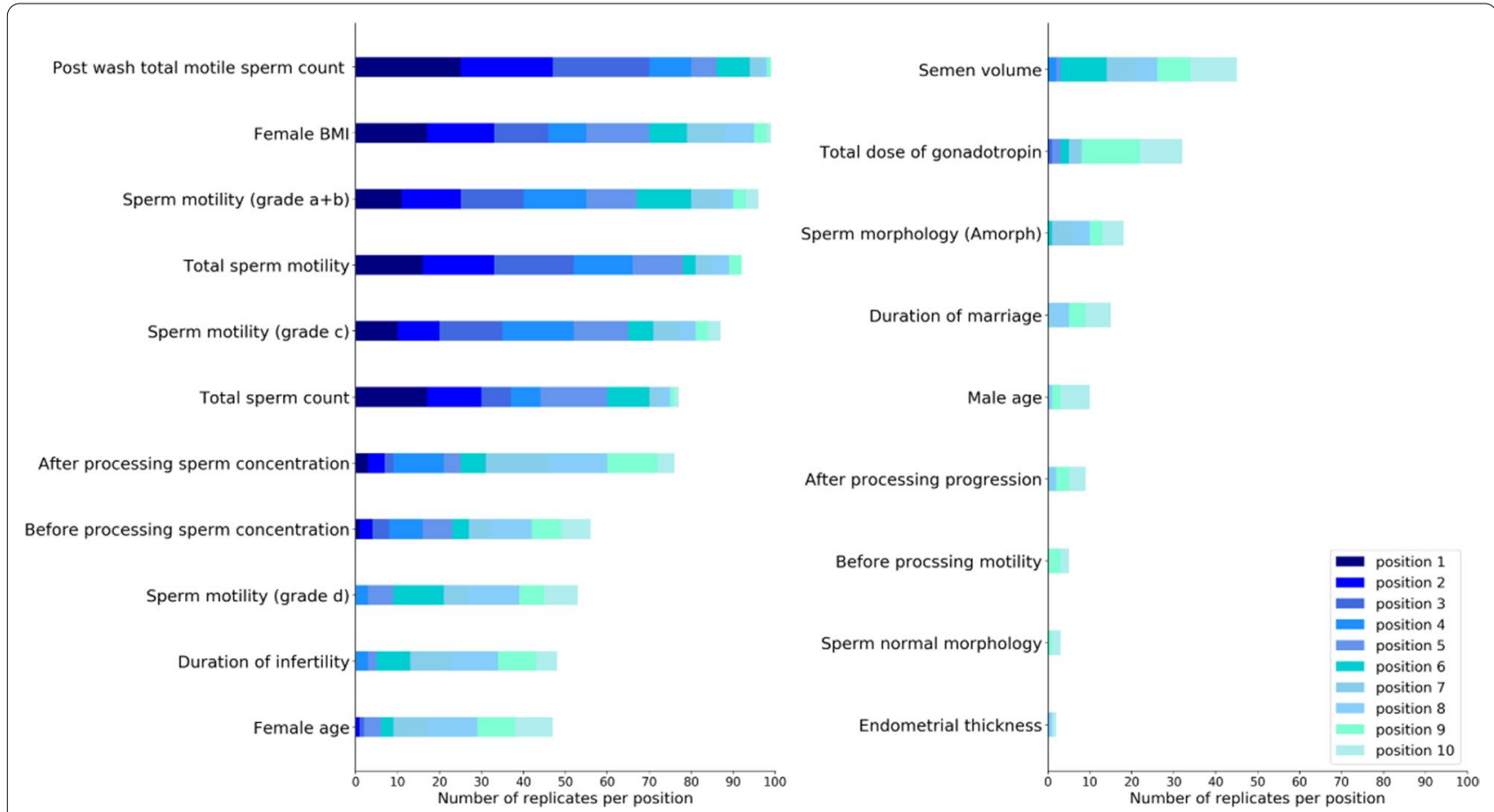

Fig. 7 Overview of top features ranked based on CNFE-SE 


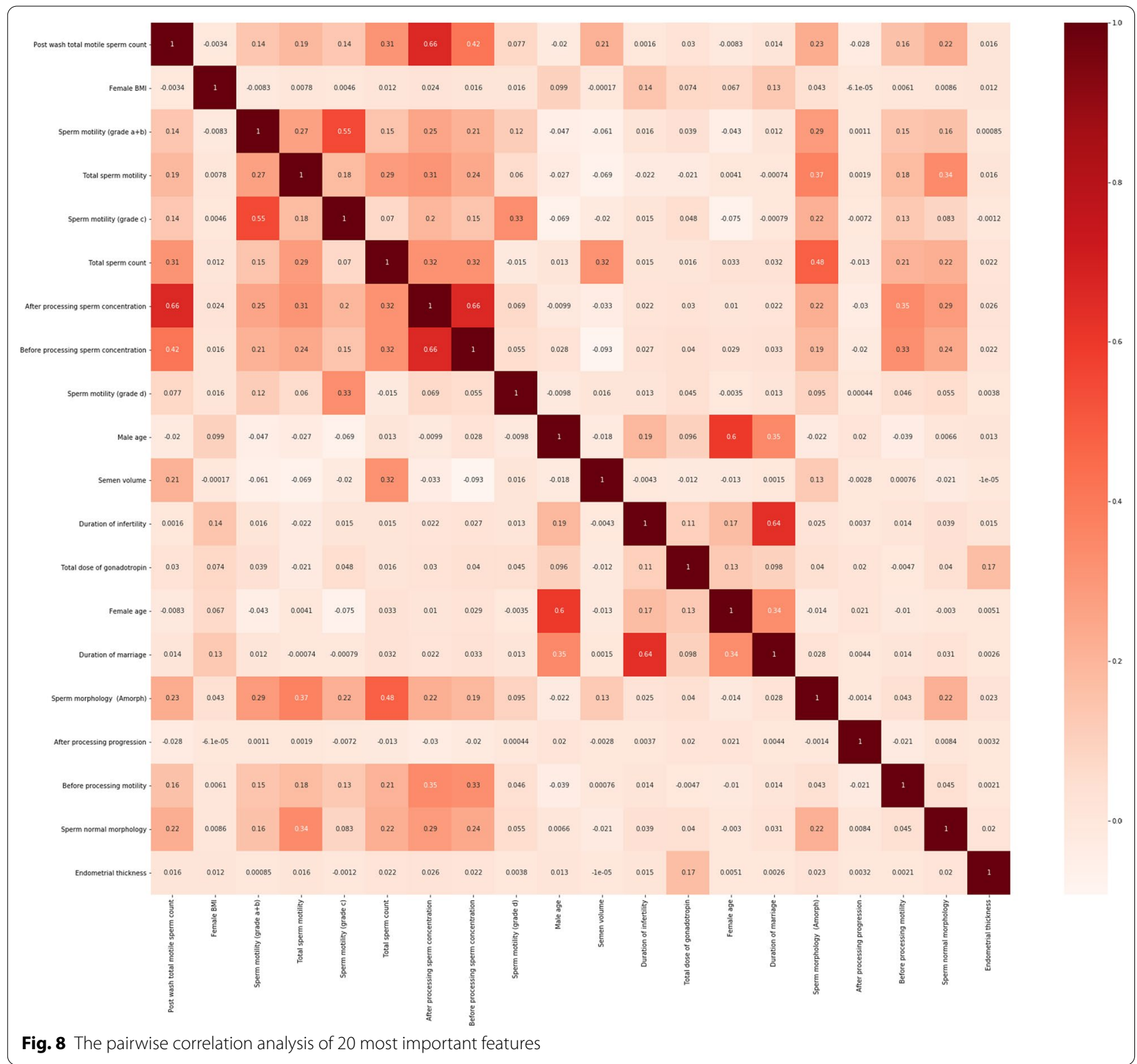

According to the results of the partial dependency plots as shown by Fig. 8, the clinical pregnancy rate has raised with increased number of post-wash total motile sperm counts and after processing sperm concentration. Also, when their values respectively vary upper than 100 million and 30 million spermatozoa per $\mathrm{ml}$, the rate of pregnancy reaches its highest rate. In addition, the likelihood of IUI success increases through growing the number of total sperm counts which is mentioned in the previous studies, too [37].

\section{Comparing the performance of CNFE-SE with other state-of-the-art classifiers}

Table 5 lists the performance measures for comparing CNFE-SE with other state of the art classifiers.

Two different feature sets are considered as the input variables fed to the classifiers including all 296 features and only the most important features (top-20 features shown in Fig. 6). Moreover, CNFE-SE is trained and evaluated twice (one time without doing feature engineering (FE) and another time with performing feature engineering).

The models are executed and trained on different random training samples up to 50 times and the 


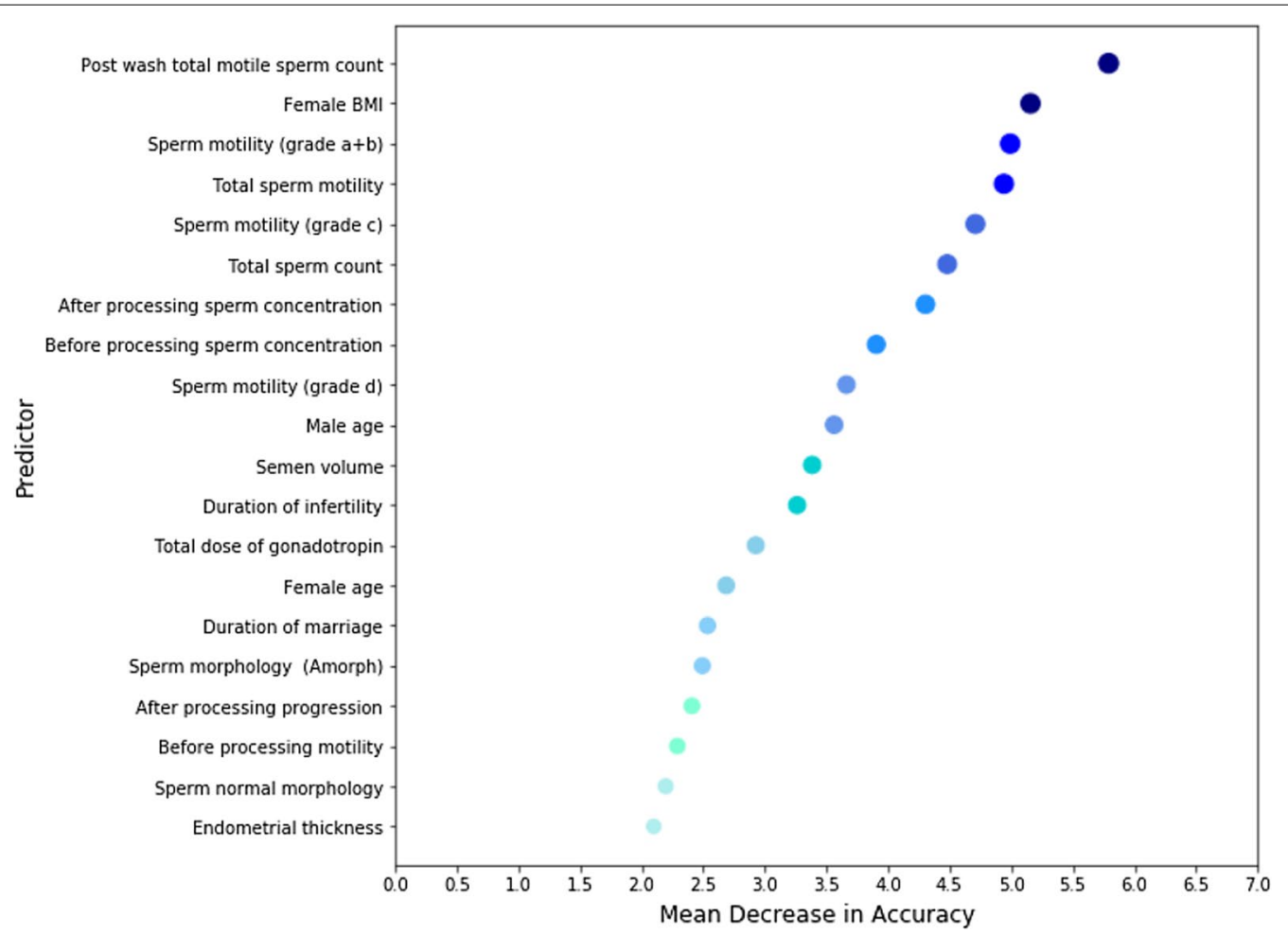

Fig. 9 MDA values of top-20 features in this study

mean \pm standard deviation values are depicted in Table 5 . The CNFE-SE outperforms the compared models by AUC of $0.84 \pm 0.01$, sensitivity of $0.79 \pm 0.01$, specificity of $0.91 \pm 0.01$, and accuracy of $0.85 \pm 0.01$ when trains on all 296 features. Moreover, CNFE-SE has the superior performance when only 20-top features are fed to it as input variables with AUC of $0.87 \pm 0.01$, sensitivity of $0.82 \pm 0.01$, specificity of $0.92 \pm 0.01$ and accuracy of $0.87 \pm 0.01$. Our obtained results show that feature engineering and considering only 20 -top features improve the performance of CNFE-SE.

Table 6 shows the confusion matrix of CNFE-SE for total dataset.

Figure 11 depicts ROC curve for CNFE-SE trained with all features.

As shown by Fig. 11, AUC of CNFE-SE trained on all features is $0.84 \pm 0.01$. As illustrated by Table 5 , the compared single classifiers show almost weak performances. The main reason is that the patients treated with IUI do not have complicated conditions and the leading cause of their infertility is idiopathic. Therefore, the data of the two classes have high similarity with each other, and their differentiation using single classifier is not an easy task. However, among these models, Light-GBM as one of state-of-the-art machine learning algorithms has the second best performance because it is a gradient boosting framework that uses tree-based learning algorithms and not only covers multi hyper-parameters but also has more focus on the accuracy of the results [35].

When the classes are imbalanced, Precision-Recall curve is a useful instrument for the presentation of prediction success. A great area under this curve shows both high precision, which is related to low false-positive rate, and high recall, refers to low false-negative rate. Figure 12 indicates the precision-recall curves for CNFE-SE trained using top-20 features.

As shown in Fig. 12, CNFE-SE predicts both classes with highly reasonable performance.

Moreover, the results of A-test method for structural risk calculation for different combinations of feature sets and classifiers are shown in Table 7.

Lower values of $\Gamma_{\zeta}^{\wedge}$ and $\Gamma_{\zeta}$ shows lower risk of the classifier for classifying previously unseen records and the higher capacity and generalization ability of the model. Therefore, the feature set and classifier achieving the lower values of $\Gamma_{\zeta} \wedge$ and $\Gamma_{\zeta}$ is more desired. As shown by Table 7, CNFE-SE trained using top-20 features has the superior performance based on A-Test results. 


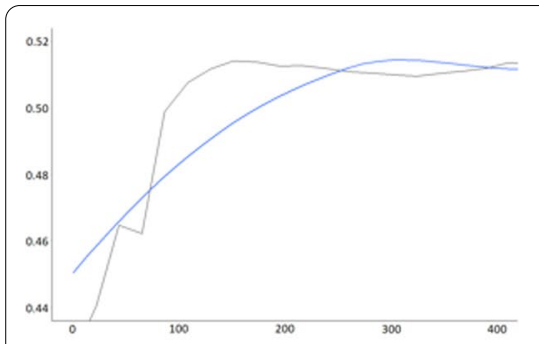

Post wash total motile sperm count $\left({ }^{*} 10^{6}\right)$

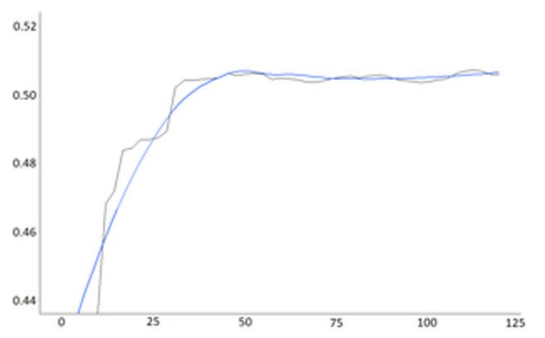

After processing sperm concentration $\left({ }^{*} 10^{6} / \mathrm{ml}\right)$

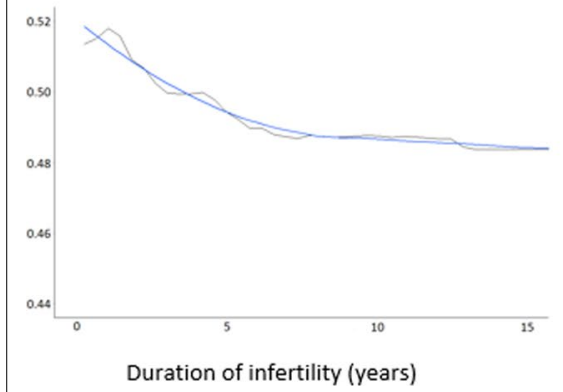

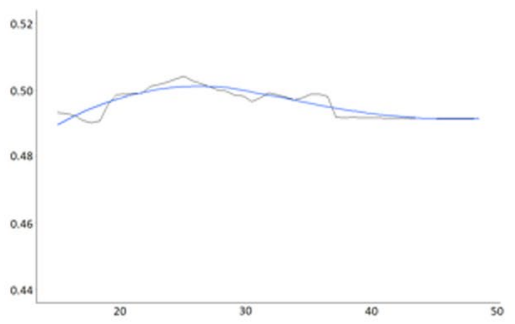

Female BMI

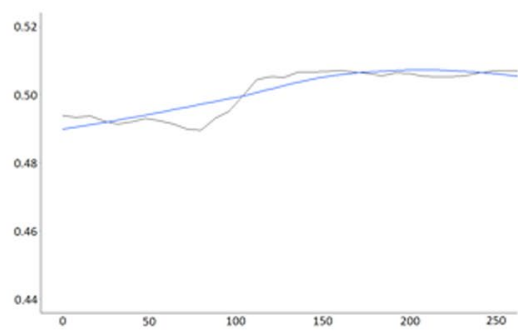

Total sperm count $\left({ }^{\star} 10^{6}\right)$

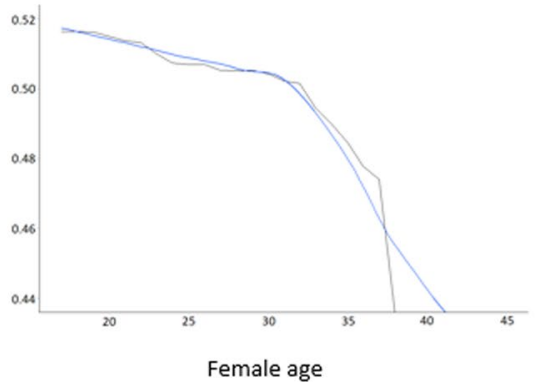

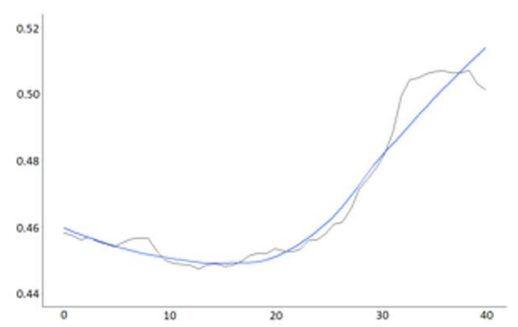

Sperm motility grade $a+b(\%)$

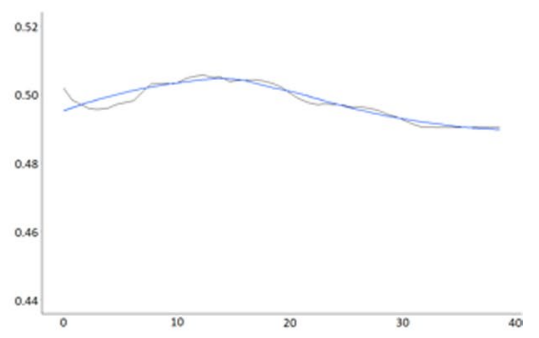

Sperm motility grade $d(\%)$

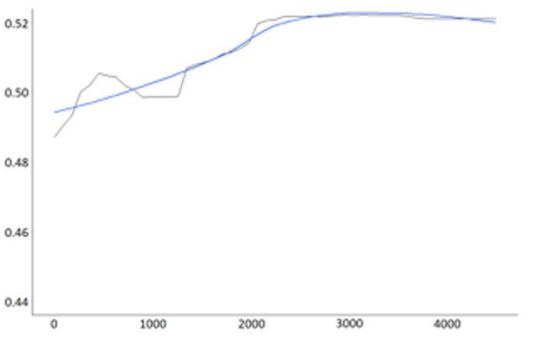

iTotal dose of gonadotropin

Fig. 10 Partial dependency plots of nine features among the important features which the blue curves indicate locally weighted smoothing. It shows pregnancy variation obtaining from CNFE-SE (y-axis) as a function of a feature ( $x$-axis) in IUI

\section{Discussion}

In the current study, among the various features that significantly affect the IUI outcome, the most potential predictors are female BMI and semen quality parameters. Semen data such as sperm count and motility are illustrated as the most prognostic factors in pregnancies, conceived by IUI and their association with IUI outcome have demonstrated in some previous studies [38]. Moreover, some previous studies have confirmed that semen descriptors, after the swim-up procedure have been more important than the ones before sperm washing process $[39,40]$. Similarly, the percentage of motile sperm and its progression in the ejaculate have been known as significant predictors in IUI outcome prediction in the literature $[41,42]$. Sperm motility grades $\mathrm{a}+\mathrm{b}$ (progressive motility) and grade d (immotile sperms) are also determined in this study as potential predictive factors for a successful IUI [43]. Thus, if their corresponding values are more than $20 \%$ and less than $15 \%$, respectively, the IUI success rate is higher.
Table 5 The confusion matrix of CNFE-SE for total dataset

\begin{tabular}{lcc}
\hline & Real positive & Real negative \\
\hline Predicted positive & 1296 & 860 \\
Predicted negative & 321 & 8772 \\
\hline
\end{tabular}

Furthermore, the results of this study indicates that the IUI success rate is almost low when the female BMI is abnormal (BMI is lower than 20 or larger than 30). If female BMI is about 25 as the normal BMI value, the probability of pregnancy increases. This finding is mentioned in the previous studies, too [44].

Previous studies have shown that pregnancy rate could be reduced by increase in the female age $[42,45]$. The present study identifies that the women older than 38 have a lower chance of successful IUI. However, Edrem et al. have not found the female age to be a prognostic factor in the prediction of IUI outcome [46]. 


\begin{tabular}{|c|c|c|c|}
\hline Feature set & Classifier & $\Gamma_{\zeta}^{\wedge}$ & Minimum of $\Gamma_{\zeta}$ \\
\hline \multirow[t]{10}{*}{ All 296 features } & RF & 26.1 & 12.8 \\
\hline & DT & 24.6 & 14.1 \\
\hline & NB & 24.9 & 12.6 \\
\hline & ANN & 25.5 & 14.5 \\
\hline & SVM & 24.7 & 20.7 \\
\hline & XGboost & 24.3 & 15.4 \\
\hline & LGBM & 24.6 & 14.2 \\
\hline & Adaboost & 25.8 & 12.7 \\
\hline & CNFE-SE without FE & 16.9 & 11.7 \\
\hline & CNFE-SE with FE & 11.3 & 6.8 \\
\hline \multirow{10}{*}{$\begin{array}{l}\text { Only most impor- } \\
\text { tant features }\end{array}$} & $\mathrm{RF}$ & 24.7 & 13.5 \\
\hline & DT & 24.3 & 14.9 \\
\hline & NB & 23.6 & 16.1 \\
\hline & ANN & 24.6 & 15.8 \\
\hline & SVM & 23.7 & 16.4 \\
\hline & XGboost & 23.1 & 15.9 \\
\hline & LGBM & 22.3 & 13.7 \\
\hline & Adaboost & 21.5 & 13.6 \\
\hline & CNFE-SE without FE & 16.1 & 11.3 \\
\hline & CNFE-SE with FE & 10.9 & 6.2 \\
\hline
\end{tabular}

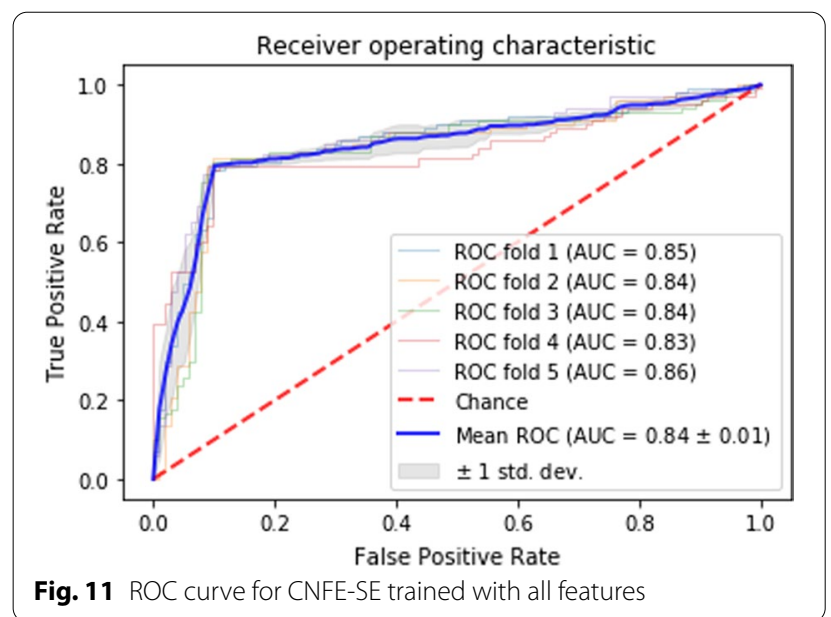

As shown in Fig. 8, the duration of infertility inversely affects the fertility rate, and the decline in fecundity is acclaimed by some previous works, as well. Also, the previous studies have shown that when the couples' duration of infertility is less than six years, the pregnancy success rate is higher [47].

The total dose of gonadotropins is taken into account in this study as an important feature. Moreover, its

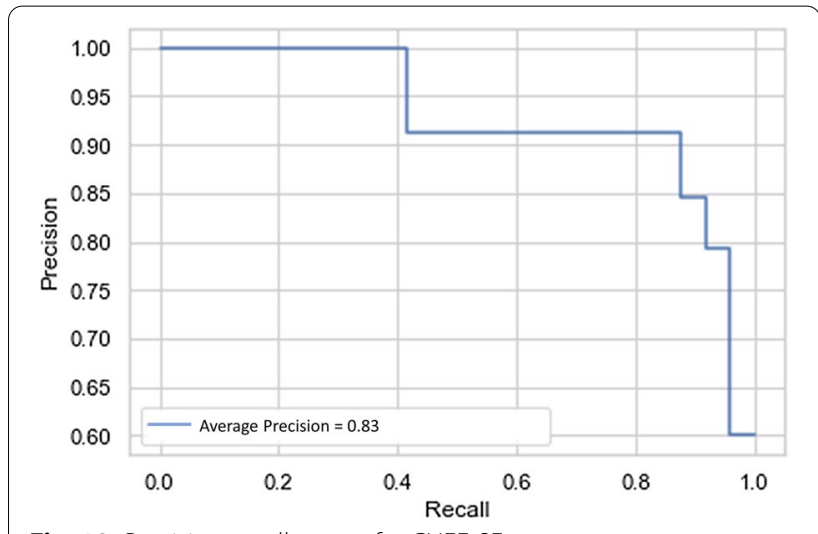

Fig. 12 Precision-recall curves for CNFE-SE

significance has been considered recently, too [11]. This study identifies that the total dose of gonadotropin is positively correlated with the pregnancy rate. Moreover, other factors contributing to failure or success of IUI outcome according to this study's findings include semen volume, male age, sperm normal and amorphous morphology, duration of the marriage, and endometrial thickness which some of them have been demonstrated as the influential attributes in some previous studies [48-50].

Eventually, the CNFE-SE is trained using the 20 most important features and it yields surprisingly good performances $(\mathrm{AUC}=0.87,95 \% \mathrm{CI} 0.86-0.88$ ). It shows that the model carried out by these features, demonstrates a highly reasonable performance.

Some studies consider different patients' cycles as independent of each other, which may lead to a biased result. For example, they have considered the first cycle information $[16,51]$. Our reanalysis of the primary cycle data revealed that the AUC performances of Light-GBM and CNFE-SE are $0.62 \pm 0.01$ and $0.84 \pm 0.01$, respectively, which does not change significantly when all the cycles are taken into account. Moreover, as shown in the materials and methods section, increasing the number of cycles augment the clinical pregnancy rate which are in line with the importance of this feature in subsequent IUI outcome $[52,53]$. On the contrary, the variable cycle number has not identified as an important feature according to CNFE-SE feature scores. This finding may be due to the high number of data in the first cycle compared to the second, third and more cycles, which approximately $74 \%$ of the data belongs to the first cycle of IUI treatment.

Finally, our study has some restrictions. Some of the female hormonal tests including FSH, TSH, LH, and AMH have not been measured in all the patients before beginning IUI cycle, and therefore they are eliminated 
Table 7 The processing time details for our proposed method (FE: Feature engineering using complex network analysis)

\begin{tabular}{llcc}
\hline Feature set & Model & Time for training (.sec) & $\begin{array}{c}\text { Time for applying } \\
\text { on one test data record } \\
\text { (.sec) }\end{array}$ \\
\hline All 296 features & CNFE-SE without FE & 1751.62 & 21.47 \\
All 296 features & CNFE-SE with FE & 1902.88 & 28.13 \\
top 20 features & CNFE-SE without FE & 619.63 & 8.05 \\
Top 20 features & CNFE-SE with FE & 744.92 & 11.26 \\
\hline
\end{tabular}

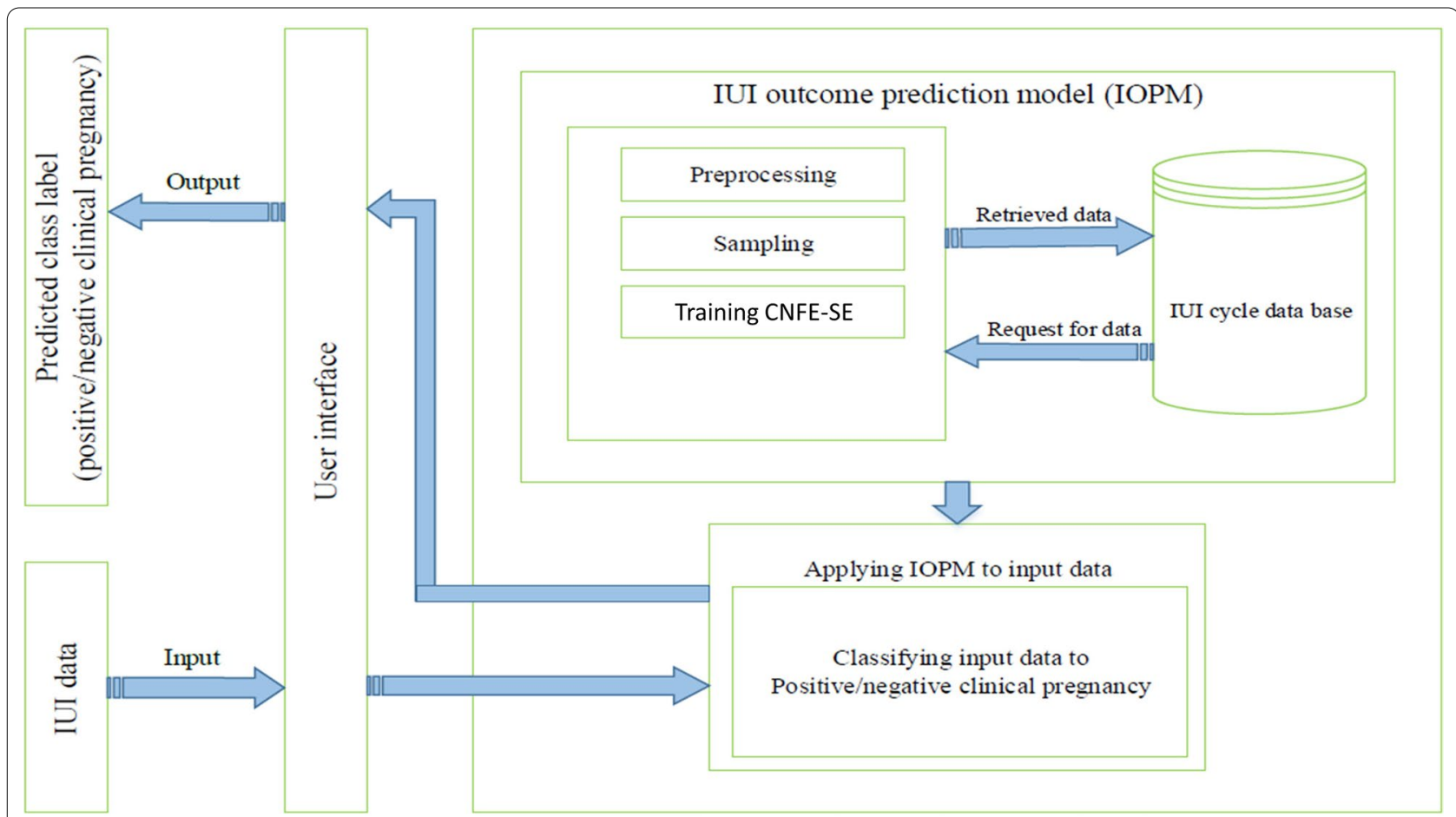

Fig. 13 Schematic of our proposed medical decision support system for IUI outcome prediction

from the analysis due to their high missing value rate. At the Royan center, the patients who are entering the IUI treatment cycles are those who do not have complicated conditions, and the women's hormonal tests are usually normal. Moreover, the male BMI is excluded because of its high rate of missing values. The features describing the geographic information of couples' habitats are removed from the study due to their low quality data entry.

Currently machine learning algorithms has been increasingly employed in different medical fields [8]. Therefore, through using machine learning methods, we are able to predict the success or failure of the IUI cycle treatment outcome for each couple, based on their demographic characteristics and cycle information. In other words, our proposed CNFE-SE model shows superior performance among the compared state of the art classifiers. A decision support system (DSS) can be designed and implemented based on CNFE-SE. This DSS can help the physicians to choose other treatment plans for the couples and reduce patients' costs if their IUI cycle success rate is low. The schematic of this medical assistance system is shown in Fig. 13.

The proposed DSS is trained on the training dataset by CNFE-SE after preprocessing the collected dataset. After completing the training of CNFE-SE, every time a new data record is registered in the DSS, it can be classified by CNFE-SE into positive or negative outcome. The predicted outcome for the new data record can assist the physicians to decide to treat the couple with IUI method or not. 


\section{Conclusion} predict the success or failure rate of the IUI could effectively improve the evaluation performances in comparison with other classical prediction models such as regression analysis. Furthermore, our proposed CNFE-SE model outperforms the compared methods with highly reasonable accuracy. CNFE-SE can be used as clinical decision-making assistance for the physicians to choose a beneficial treatment plan with regards to their patients' therapy options, which would reduce the patients' costs as well.

The experimental results in this study show that the most important features for predicting IUI outcome are semen parameters (sperm motility and concentration) as well as female BMI.

Some features which have been identified as good discriminative features for IUI outcome prediction in the previous studies are excluded from this study because of their high missing value rate. For example, some of the female hormonal tests including FSH, TSH, LH, and $\mathrm{AMH}$ are not routinely measured in all the patients before IUI and they are excluded from the study. It is proposed to augment dataset with data records without missing value in the mentioned features and consider the excluded features to CNFE-SE, and then try to rank the augmented feature set and evaluate the performance of the classifier.

On the other hand, some data records have noisy information which can reduce the performance of the classifiers. As future work, it is suggested that improving the robustness of CNFE-SE against the noisy data by including vote-boosting and other previously proposed methods for increasing the noise robustness of the classifiers. Moreover, the data is highly imbalanced which can have negative effect on the classifiers' performance. As another research opportunity, it is suggested that reducing the influence of data distribution per class by incorporating the advanced balanced sampling strategies.

Determining the optimal ensemble size is a challenging issue, yet. It is suggested that the impact of the ensemble size on the overall performance of stacked ensemble is studied in the future studies on different tasks and different datasets.
In conclusion, the use of machine learning methods to

ensemble; C.V.: Cross validation; DSS: Decision support system; DT: Decision tree; FN: False negative; FP: False Positive; FSH: Follicle-stimulating hormone; HCA: Hierarchical clustering analysis; ICSI: Intracytoplasmic injection; IUI: Intrauterine Insemination; IVF: In-vitro fertilization (IVF); K-NN: K-nearest neighbors; LH: Luteinizing Hormone; LR: Logistic regression; MDA: Mean decrease of accuracy; MLP: Multi-layer perceptron; N: Negative; NB: Naïve Bayes; P: Positive; PCA: Principal component analysis; RF: Random forest; RBF: Radial basis function; SVM: Support vector machines; SD: Standard deviation; TN: True negative; TP: True positive; TSH: Thyroid-stimulating hormone.

\section{Acknowledgements}

The authors acknowledge the Royan institute staffs, especially the informatics department for their valuable contributions. There is no conflict of interest in this study.

\section{Authors' Contributions}

Conceptualization: SR, TK and MT. Data curation: SR, TK and MT. Formal analysis: SR, TK and MT. Funding acquisition: there is no funding. Investigation: SR, TK and MT. Methodology: SR and TK. Project administration: TK. Software: SR and TK. Supervision: TK and MT. Validation: SR, TK, AVTD, HS, MT, FG. Visualization: SR, TK and MT. Writing - original draft: SR and TK. Writing - review \& editing: SR, TK, AVTD, HS, MT, FG. All authors have read and approved the manuscript

\section{Funding}

This study was not funded by any organization.

\section{Availability of data and materials}

Our study is a retrospective study of a 5-year couples' data undergoing IUI. Data is collected from Reproductive Biomedicine Research Center, Royan Institute for 8,360 couples who underwent 11,255 IUI cycles were included. But, we are not allowed to share the original dataset because of the privacy and security issues.

\section{Ethics approval and consent to participate}

This study is approved by the institutional review board of the ROYAN Institute (IR.ACECR.ROYAN.REC.1398.213). The informed consent requirement for this study was waived because this was a retrospective study with little patients' sensitive or personal information, and all data were anonymized. The full name of the ethics committee who approved this study is IR.ACECR.ROYAN. REC which ROYAN Institute belongs to. The committee's reference number is IR.ACECR.ROYAN.REC.1398.213.

\section{Consent for publication}

Not applicable.

\section{Competing interests}

The authors declare that there are no conflicts of interest.

\section{Author details}

1 School of Industrial and Systems Engineering, Tarbiat Modares University, Tehran, Iran. ${ }^{2}$ Department of Genetics At Reproductive Biomedicine Research Center, Royan Institute for Reproductive Biomedicine, ACECR, Tehran, Iran. ${ }^{3}$ Department of Reproductive Imaging, Reproductive Biomedicine Research Center, Royan Institute for Reproductive Biomedicine, ACECR, Tehran, Iran.

${ }^{4}$ Department of Andrology, Reproductive Biomedicine Research Center, Royan Institute for Reproductive Biomedicine, ACECR, Tehran, Iran. ${ }^{5}$ Department of Endocrinology and Female Infertility, Reproductive Biomedicine Research Center, Royan Institute for Reproductive Biomedicine, ACECR, Tehran, Iran.

\section{Appendix}

ACECR: Academic center for education, culture and research; AMH: Antimüllerian hormone; ANN: Artificial neural networks; ART: Assisted reproductive technology; AUC: Area under curve; BMI: Body mass index; CN1: Complex network which is comprised of all the training data records as its nodes; CN2: Complex network which includes all training data excluding negative class; CN3: Complex network which includes all training data excluding positive class; CNFE-SE: Complex network-based feature engineering and stacked
See Tables 8 and 9. 
Table 8 Feature description for our dataset

\begin{tabular}{|c|}
\hline Variable \\
\hline Endoscopy-hysteroscopy \\
\hline Endoscopy-laparoscopy \\
\hline Endocrine disorder-pituitary \\
\hline History-Habit \\
\hline Medical HX-neuro psychological \\
\hline Medical HX-Gl \\
\hline Medical HX-immunologic disorder \\
\hline Medical HX-respiratory disease \\
\hline Common surgery-orchiopexy \\
\hline Common surgery-varicocelectomy \\
\hline Common surgery-hernia \\
\hline Common surgery-hydrocele \\
\hline History-nipple discharge \\
\hline Current drug-antihyperprolactinemia \\
\hline Medical HX-infection disorder \\
\hline Endocrine disorder-thyroid \\
\hline Medical HX cardiovascular disorder \\
\hline Menstrual criteria-with drug \\
\hline History-pelvic infection HX \\
\hline Current drug-antidiabetic \\
\hline
\end{tabular}

LTG
RT G__
LT G_1

Medical HX-blood disorder

Common surgery-orchiectomy

Current drug-thyroid drugs

Past-irreg

Medical HX-renal disease

Diabetes-1st degree relative

Endocrine disorder-D.M

diagnosis-Uterine $\mathrm{F}$

diagnosis-ovulatory $\mathrm{F}$

Therapeutic-agglutination

specimen characteristics-color

Therapeutic-type of sampling

Therapeutic sperm preparation -technique

diagnosis-recurrent abortion

Tuboperitoneal type

diagnosis-unexplained

diagnosis-other diagnosis

Menstrual criteria-amount

IUI clinic-IUI catheter

\section{Variable levels}

Variable type

0, 1st Hysteroscopy, 1st Hysteroscopy | 2nd Hysteroscopy, 1st Hysteroscopy Nominal | 2nd Hysteroscopy | 3rd Hysteroscopy, 3rd Hysteroscopy | 1st Hysteroscopy, 4th Hysteroscopy | 3rd Hysteroscopy | 1st Hysteroscopy | 2nd Hysteroscopy

0, 1st laparoscopy, 1st laparoscopy | 2nd laparoscopy, 2nd laparoscopy

0 , acromegaly, Prolactinoma

0, Alcohol, Alcohol | smoking, smoking

0, Anxiety, depression, depression | Epilepsy, Epilepsy, Psychotic disorder

0, Appendicitis, IBD, IBS, IBS | Peptic ulcer, Peptic ulcer

0 , Arthritic rheumatoid, lupus

0, Asthma, Bronchiectasis, bronchitis, bronchitis | Asthma

0, Bilat, Bilat | Left | Right, Left, Right, Right | Left

0, Bilat, Left, Left | Bilat | Right, Left | Right, Right

0, Bilat, Left, Left | Right, Left | Right | Bilat, Right

0 , Bilat, Left, Right

0, Bilateral, Bloody, Unilateral

0, Bromocriptin, Bromocriptin | Dostinex, Dostinex

0, Brucellosis, Hepatitis A, Hepatitis B, Hepatitis C, Herpes, TB

0, cancer, Hyper, Hyper | Hypo, Hypo, Nodule

0, DVT, Heart failure, Hypertension, Hypertension | MVP, MVP

0 , Estrogen, OCP, Progesterone, Progesterone | Estrogen, Progesterone | OCP

0 , Frequent discharge, History Of PID

0, Glucophage, Metformin, Metformin | Glucophage, Metformin | Paraovarian cyst, Paraovarian cyst

0, II, I| | III, I| | Time of Injection, III, Time of Injection

Nominal

Nominal

Nominal

Nominal

Nominal

Nominal

Nominal

Nominal

Nominal

Nominal

Nominal

Nominal

Nominal

Nominal

Nominal

Nominal

Nominal

Nominal

Nominal

Nominal

0, II, II | Time of Injection, Time of Injection

Nominal

0, III, Time of Injection

0 , Iron anemia, Major thalassemia, minor thalassemia

0 , Left, Right

0 , Levothyroxin, Levothyroxin | metimazole, metimazole, PTO

Nominal

Nominal

Nominal

Nominal

Nominal

Nominal

0,Recurrent infection, Renal anomaly, Renal stone

Nominal

Nominal

0, type1, type2

adenomyosis, Anomaly, Asherman, Myoma, Negative, Unexplained (thin endometrium)

Age factor, Diminished ovarian reserve, Endocrine problem, Hypothalamic hypogonadism, Negative, PCOS

all, few, most, none, some

Amber-Yellow, gray | yellow, Grey, Light-Yellow, Milky, White, White-Grey

Coitus, Coitus | Masturbation, Masturbation, Retrograde ejaculation, Retrograde ejaculation | Coitus

Discontinuous gradient, Pure sperm | Discontinuous gradient, Swim up, Nominal Swim up | Discontinuous gradient

Endocrine, Male, Negative, Thrombotic, unexplained

Endometriosis, EP Hx, hydrosalipinx, Idiopathic, Negative, Post surgery

Endometriosis, Idiopathic, Negative

Nominal

Nominal

Nominal

Nominal

Nominal

Nominal

Nominal

Nominal

Genetic, Impotency, Incomplete data, Negative, other, poor obstetric Nominal outcome, sex selection, Vaginismus

hypermenorrhea, Hypo menorrhea, Normal

Nominal

INDOVASIVE, Other, INDOVASIVE | Other, Catheter Impex, ORI 
Table 8 (continued)

\begin{tabular}{|c|c|c|}
\hline Variable & Variable levels & Variable type \\
\hline Past-type & more than 8 levels & Nominal \\
\hline Surgical HX-non gynecologic surgery & more than 8 levels & Nominal \\
\hline DRUG HX-past drug & more than 8 levels & Nominal \\
\hline Male medical information-exposures & more than 8 levels & Nominal \\
\hline Male medical information-drug Hx esp & more than 8 levels & Nominal \\
\hline Coded after processing-progression & more than 8 levels & Nominal \\
\hline Coded before processing-progression & more than 8 levels & Nominal \\
\hline Therapeutic-viscosity & Normal, Somewhat Thick, Thick, Thin, Very thick & Nominal \\
\hline Specimen characteristics-viscosity & Normal, Somewhat Thick, Thick, Very Thick & Nominal \\
\hline Family history endocrine disorder-thyroid & NO, YES & Binominal \\
\hline Male medical information-scrotum & $\mathrm{Ab}, \mathrm{NL}$ & Binominal \\
\hline Specimen characteristics-collect type & $C, M$ & Binominal \\
\hline Therapeutic-place of sampling & In, Out & Binominal \\
\hline Therapeutic-SPLIT Ejaculation & $\mathrm{NO}, \mathrm{YES}$ & Binominal \\
\hline Menstrual Criteria-Interval & Normal ( $22-35$ days), Abnormal ( $<21,>36$ days) & Binominal \\
\hline Infertility type & primary, secondary & Binominal \\
\hline Relationship-First cousin & 0,1 & Binary \\
\hline Relationship-distant relationship & 0,1 & Binary \\
\hline No relationship & 0,1 & Binary \\
\hline Diagnosis-Male factor & 0,1 & Binary \\
\hline Past-Reg (menstruation) & 0,1 & Binary \\
\hline Current-Reg (menstruation) & 0,1 & Binary \\
\hline Irreg-Oligomenorrhea & 0,1 & Binary \\
\hline Irreg-Poly menorrhea & 0,1 & Binary \\
\hline Cycle-Amenorrhea & 0,1 & Binary \\
\hline Amenorrhea-Primary & 0,1 & Binary \\
\hline Amenorrhea-Secondary & 0,1 & Binary \\
\hline Menstrual Criteria-IMB & 0,1 & Binary \\
\hline Coital HX-Lubricant & 0,1 & Binary \\
\hline Coital HX-PCB & 0,1 & Binary \\
\hline Coital HX-Vaginismus & 0,1 & Binary \\
\hline Coital HX-Impotency & 0,1 & Binary \\
\hline History-Cytotoxic Therapy & 0,1 & binary \\
\hline Allergy-food & 0,1 & Binary \\
\hline Allergy-seasonal & 0,1 & Binary \\
\hline Allergy-Skin & 0,1 & Binary \\
\hline Allergy-respiratory & 0,1 & Binary \\
\hline Family history-infertility & 0,1 & Binary \\
\hline Family history-recurrent abortion & 0,1 & Binary \\
\hline Family history-POF & 0,1 & Binary \\
\hline Family history-hearing disorder & 0,1 & Binary \\
\hline Family history-TB & 0,1 & Binary \\
\hline Family history-mental retardation & 0,1 & Binary \\
\hline Cardio vascular-myocardial infarction & 0,1 & Binary \\
\hline Cardio vascular-HTN & 0,1 & Binary \\
\hline Respiratory disorder-Asthma & 0,1 & Binary \\
\hline Thalassemia-minor & 0,1 & Binary \\
\hline Type of cancer-breast cancer & 0,1 & Binary \\
\hline Type of cancer-colon cancer & 0,1 & Binary \\
\hline
\end{tabular}


Table 8 (continued)

\begin{tabular}{|c|c|c|}
\hline Variable & Variable levels & Variable type \\
\hline Type of cancer-leukemia & 0,1 & Binary \\
\hline Type of cancer-uterus cancer & 0,1 & Binary \\
\hline Type of cancer-lung cancer & 0,1 & Binary \\
\hline Family history-epilepsy & 0,1 & Binary \\
\hline Antihyperprolactinemia-Bromocriptin & 0,1 & Binary \\
\hline Antihyperprolactinemia-Dostinex & 0,1 & Binary \\
\hline Current drug-Anti Depression & 0,1 & Binary \\
\hline Current drug-Anti hypertension & 0,1 & Binary \\
\hline Current drug-Anticoagulant & 0,1 & Binary \\
\hline Current drug-Folic acid & 0,1 & Binary \\
\hline Current drug-Estrogen & 0,1 & Binary \\
\hline Current drug-Progestron & 0,1 & Binary \\
\hline Current drug-Ferrous sulphate & 0,1 & Binary \\
\hline Current drug-Multi vitamin & 0,1 & Binary \\
\hline Physical Exam-hirsutism & 0,1 & Binary \\
\hline Type of pregnancy-Clinical pregnancy (class variable) & 0,1 & Binary \\
\hline Minimal stimulation-Gonadotropin & 0,1 & Binary \\
\hline Minimal stimulation-Letrosol & 0,1 & Binary \\
\hline Minimal stimulation-Clomiphen & 0,1 & Binary \\
\hline Type-Cetrorelix & 0,1 & Binary \\
\hline HCG.IU_5000 & 0,1 & Binary \\
\hline HCG.IU_10000 & 0,1 & Binary \\
\hline HCG.IU_15000 & 0,1 & Binary \\
\hline Buserelin_0.5 cc & 0,1 & Binary \\
\hline Ovitrelle_250 mgr & 0,1 & Binary \\
\hline Ovitrelle_500 mgr & 0,1 & Binary \\
\hline Type-Fostimon & 0,1 & Binary \\
\hline Type-Gonal.F & 0,1 & Binary \\
\hline Type-Menopour & 0,1 & Binary \\
\hline Type-HMG & 0,1 & Binary \\
\hline Type-Merional & 0,1 & Binary \\
\hline Type-Bravelle & 0,1 & Binary \\
\hline Before Stimulation-OCP & 0,1 & Binary \\
\hline Before Stimulation-Estradiol & 0,1 & Binary \\
\hline Cotreatment-Aspirin & 0,1 & Binary \\
\hline Adjuvant therapy-Acid folic & 0,1 & Binary \\
\hline Right-PCO & 0,1 & Binary \\
\hline Right-Cyst & 0,1 & Binary \\
\hline Left-PCO & 0,1 & Binary \\
\hline Left-Cyst & 0,1 & Binary \\
\hline Endometrial Texture-Three line & 0,1 & Binary \\
\hline have you married before-yes & 0,1 & Binary \\
\hline did you have any child-no & 0,1 & Binary \\
\hline Common surgery-T.BX & 0,1 & Binary \\
\hline Common surgery-Vasectomy & 0,1 & Binary \\
\hline Uncommon surgery-Urethral stricture & 0,1 & Binary \\
\hline Uncommon surgery-Brain surgey & 0,1 & Binary \\
\hline Medical history-Mumps & 0,1 & Binary \\
\hline Medical history-Hypertension & 0,1 & Binary \\
\hline
\end{tabular}


Table 8 (continued)

\begin{tabular}{|c|c|c|}
\hline Variable & Variable levels & Variable type \\
\hline Medical history-Veneral Dz & 0,1 & Binary \\
\hline Medical history-Allergy & 0,1 & Binary \\
\hline Medical history-D.M & 0,1 & Binary \\
\hline Medical history-M orchitis & 0,1 & Binary \\
\hline Medical history-Test Pain & 0,1 & Binary \\
\hline Medical history-TB & 0,1 & Binary \\
\hline Medical history-Epididimorchitis & 0,1 & Binary \\
\hline Medical history-UDT & 0,1 & Binary \\
\hline Medical history-UTI & 0,1 & Binary \\
\hline Cigarette-occasionally & 0,1 & Binary \\
\hline Cigarette-quarter pack per day & 0,1 & Binary \\
\hline Cigarette-half pack per day & 0,1 & Binary \\
\hline Cigarette-one pack per day & 0,1 & Binary \\
\hline Cigarette-two packs per day & 0,1 & Binary \\
\hline Cigarette-three packs per day & 0,1 & Binary \\
\hline Alcohol-occasionally & 0,1 & Binary \\
\hline Alcohol-one per week & 0,1 & Binary \\
\hline Opium-occasionally & 0,1 & Binary \\
\hline Opium-one per day & 0,1 & Binary \\
\hline Marital status of brothers-Married & 0,1 & Binary \\
\hline Marital status of brothers-New couples & 0,1 & Binary \\
\hline Family HX of infertility-Do your brothers have any children & 0,1 & Binary \\
\hline Right testicle Size_less than 1 & 0,1 & Binary \\
\hline Right testicle Size_1-1.5 & 0,1 & Binary \\
\hline Right testicle Size_1.5-2 & 0,1 & Binary \\
\hline Right testicle Size_2-2.5 & 0,1 & Binary \\
\hline Right testicle Size_2.5-3 & 0,1 & Binary \\
\hline Right testicle Size_3-3.5 & 0,1 & Binary \\
\hline Right testicle Size_3.5-4 & 0,1 & Binary \\
\hline Right testicle Size_more than 4 & 0,1 & Binary \\
\hline Left testicle size_less than 1 & 0,1 & Binary \\
\hline Left testicle size_1-1.5 & 0,1 & Binary \\
\hline Left testicle size_1.5-2 & 0,1 & Binary \\
\hline Left testicle size_2-2.5 & 0,1 & Binary \\
\hline Left testicle size_2.5-3 & 0,1 & Binary \\
\hline Left testicle_3-3.5 & 0,1 & Binary \\
\hline Left testicle size_3.5-4 & 0,1 & Binary \\
\hline Left testicle size_more than 4 & 0,1 & Binary \\
\hline Vas-RT & 0,1 & Binary \\
\hline Vas-LT & 0,1 & Binary \\
\hline RT-NL & 0,1 & Binary \\
\hline RT-Ab & 0,1 & Binary \\
\hline LT-NL & 0,1 & Binary \\
\hline RT-G & 0,1 & Binary \\
\hline plan-med treatment & 0,1 & Binary \\
\hline Vitamin-Vitamin C & 0,1 & Binary \\
\hline Vitamin-Vitamin E & 0,1 & Binary \\
\hline Plan-S.A & 0,1 & Binary \\
\hline Plan-Imaging & 0,1 & Binary \\
\hline
\end{tabular}


Table 8 (continued)

\begin{tabular}{|c|c|c|}
\hline Variable & Variable levels & Variable type \\
\hline Plan-H.A & 0,1 & Binary \\
\hline Plan-CW & 0,1 & Binary \\
\hline Plan-KARYO & 0,1 & Binary \\
\hline Plan-AZF & 0,1 & Binary \\
\hline Plan-TESE & 0,1 & Binary \\
\hline Plan-Wives visit by Gyn required & 0,1 & Binary \\
\hline Plan-Varicocelectomy & 0,1 & Binary \\
\hline Plan-sperm Freezing & 0,1 & Binary \\
\hline Plan-PC.U.A & 0,1 & Binary \\
\hline Plan-genetic consult & 0,1 & Binary \\
\hline Plan-endocrine consult & 0,1 & Binary \\
\hline Plan-DFI & 0,1 & Binary \\
\hline Plan-stop smoking, alcohol, opium & 0,1 & Binary \\
\hline Plan-Occupation hygiene & 0,1 & Binary \\
\hline Plan-Low weight & 0,1 & Binary \\
\hline Plan-Others & 0,1 & Binary \\
\hline Morphology-Amorph & 0,1 & Binary \\
\hline Morphology-giant head & 0,1 & Binary \\
\hline Morphology-pin head & 0,1 & Binary \\
\hline Morphology-round head & 0,1 & Binary \\
\hline Morphology-cytoplasmic droplet & 0,1 & Binary \\
\hline
\end{tabular}


Table 9 Summary statistics for the features of our dataset

\begin{tabular}{|c|c|c|c|c|c|c|c|}
\hline Feature name & Min & First Q & Median & Third Q & Max & Mean & SD \\
\hline \multirow[t]{2}{*}{ Time diff between cycles } & 0 & 0 & 0 & 2 & 6.1 & 1.59 & 4.82 \\
\hline & 0 & 0 & 0 & 1 & 6.9 & 1.25 & 3.83 \\
\hline \multirow[t]{2}{*}{ Age_x } & 17 & 25 & 28 & 31 & 43 & 28.29 & 4.29 \\
\hline & 16 & 26 & 29 & 32 & 47 & 28.97 & 4.68 \\
\hline \multirow[t]{2}{*}{ Family demographic information (Education_x) } & 1 & 3 & 3 & 5 & 7 & 3.43 & 1.17 \\
\hline & 1 & 3 & 3 & 5 & 7 & 3.4 & 1.22 \\
\hline \multirow[t]{2}{*}{ Duration of marriage (years) } & 0.42 & 4 & 6 & 7 & 23 & 6 & 2.94 \\
\hline & 0.25 & 4 & 6 & 7.5 & 26 & 6.31 & 3.19 \\
\hline \multirow[t]{2}{*}{ Duration of infertility (Years) } & 0.25 & 2.5 & 4 & 5 & 15 & 4.36 & 2.62 \\
\hline & 0.17 & 2.84 & 4.5 & 6 & 19 & 4.66 & 2.85 \\
\hline \multirow[t]{2}{*}{ Menstrual HX (menarche old year) } & 9 & 13 & 13 & 14 & 27 & 13.35 & 1.46 \\
\hline & 9 & 13 & 13 & 14 & 27 & 13.29 & 1.42 \\
\hline \multirow[t]{2}{*}{ Current contraception duration } & 0 & 0 & 0 & 0 & 3 & 0.02 & 0.17 \\
\hline & 0 & 0 & 0 & 0 & 8 & 0.01 & 0.19 \\
\hline \multirow[t]{2}{*}{ Summary-Gravida } & 0 & 0 & 0 & 0 & 6 & 0.29 & 0.66 \\
\hline & 0 & 0 & 0 & 0 & 6 & 0.26 & 0.61 \\
\hline \multirow[t]{2}{*}{ Summary-para } & 0 & 0 & 0 & 0 & 3 & 0.08 & 0.31 \\
\hline & 0 & 0 & 0 & 0 & 4 & 0.08 & 0.3 \\
\hline \multirow[t]{2}{*}{ Abortion-early } & 0 & 0 & 0 & 0 & 4 & 0.17 & 0.48 \\
\hline & 0 & 0 & 0 & 0 & 6 & 0.15 & 0.45 \\
\hline \multirow[t]{2}{*}{ Abortion-late } & 0 & 0 & 0 & 0 & 3 & 0.02 & 0.16 \\
\hline & 0 & 0 & 0 & 0 & 4 & 0.01 & 0.11 \\
\hline \multirow[t]{2}{*}{ Summary-IUFD } & 0 & 0 & 0 & 0 & 2 & 0.01 & 0.11 \\
\hline & 0 & 0 & 0 & 0 & 2 & 0 & 0.07 \\
\hline \multirow[t]{2}{*}{ Summary-mole } & 0 & 0 & 0 & 0 & 1 & 0 & 0.06 \\
\hline & 0 & 0 & 0 & 0 & 2 & 0 & 0.04 \\
\hline \multirow[t]{2}{*}{ Summary-EP } & 0 & 0 & 0 & 0 & 1 & 0.01 & 0.11 \\
\hline & 0 & 0 & 0 & 0 & 1 & 0.02 & 0.12 \\
\hline \multirow[t]{2}{*}{ Summary-Preterm } & 0 & 0 & 0 & 0 & 3 & 0.03 & 0.2 \\
\hline & 0 & 0 & 0 & 0 & 5 & 0.02 & 0.16 \\
\hline \multirow[t]{2}{*}{ Summary-term } & 0 & 0 & 0 & 0 & 2 & 0.06 & 0.25 \\
\hline & 0 & 0 & 0 & 0 & 4 & 0.06 & 0.26 \\
\hline \multirow[t]{2}{*}{ Summary-living child } & 0 & 0 & 0 & 0 & 2 & 0.06 & 0.24 \\
\hline & 0 & 0 & 0 & 0 & 4 & 0.06 & 0.25 \\
\hline \multirow[t]{2}{*}{ IUI Hx-total } & 2 & 2 & 2 & 3 & 10 & 2.73 & 1.47 \\
\hline & 2 & 2 & 2 & 3 & 11 & 2.7 & 1.4 \\
\hline \multirow[t]{2}{*}{ Result-failed } & 0 & 0 & 0 & 0.41 & 6 & 0.39 & 0.78 \\
\hline & 0 & 0 & 0 & 1 & 10 & 0.41 & 0.78 \\
\hline \multirow[t]{2}{*}{ Result-pregnant } & 0 & 0 & 0 & 0 & 2 & 0.04 & 0.2 \\
\hline & 0 & 0 & 0 & 0 & 2 & 0.02 & 0.14 \\
\hline Summary of ART cycle-IVF & 0 & 0 & 0 & 0 & 2 & 0.01 & 0.13 \\
\hline & 0 & 0 & 0 & 0 & 6 & 0.03 & 0.24 \\
\hline Summary of ART cycle-ZIFT & 0 & 0 & 0 & 0 & 1 & 0 & 0.02 \\
\hline & 0 & 0 & 0 & 0 & 3 & 0 & 0.06 \\
\hline Summary of ART cycle-E.T freeze & 1 & 1 & 1 & 1 & 4 & 1.01 & 0.11 \\
\hline & 1 & 1 & 1 & 1 & 5 & 1.01 & 0.1 \\
\hline Summary of ART cycle-Total & 0 & 0 & 0 & 0 & 4 & 0.02 & 0.2 \\
\hline & 0 & 0 & 0 & 0 & 9 & 0.04 & 0.31 \\
\hline Physical exam-height & 142 & 158 & 161.05 & 165 & 187 & 161.61 & 5.74 \\
\hline
\end{tabular}


Table 9 (continued)

\begin{tabular}{|c|c|c|c|c|c|c|c|}
\hline Feature name & Min & First Q & Median & Third Q & Max & Mean & SD \\
\hline & 142 & 157 & 161.05 & 164 & 187 & 160.96 & 5.7 \\
\hline \multirow[t]{2}{*}{ Physical exam-weight } & 40 & 61 & 67.31 & 74 & 170 & 68.14 & 12.54 \\
\hline & 36 & 60 & 67 & 73 & 171 & 67.18 & 11.65 \\
\hline \multirow[t]{2}{*}{ Physical exam-BMl } & 15.02 & 23.53 & 25.93 & 28.04 & 52.11 & 26.01 & 4.23 \\
\hline & 14.19 & 23.34 & 25.93 & 28.01 & 57.09 & 25.92 & 4.22 \\
\hline \multirow[t]{2}{*}{ IUI clinic-number of cycle } & 1 & 1 & 1 & 2 & 5 & 1.35 & 0.63 \\
\hline & 1 & 1 & 1 & 2 & 7 & 1.31 & 0.59 \\
\hline \multirow[t]{2}{*}{ Infertility medical treatment-Starting day of Stimulation (menstrual day) } & 1 & 3 & 3 & 3.42 & 8 & 3.39 & 0.73 \\
\hline & 1 & 3 & 3 & 3.42 & 14 & 3.42 & 0.82 \\
\hline \multirow[t]{2}{*}{ Letrosol dose (mg/day) } & 0 & 0 & 0 & 1.12 & 7.5 & 1.28 & 2.04 \\
\hline & 0 & 0 & 0 & 1.12 & 7.5 & 1.1 & 1.9 \\
\hline \multirow[t]{2}{*}{ Letrosol duration } & 0 & 0 & 0 & 1.19 & 12 & 1.34 & 2.17 \\
\hline & 0 & 0 & 0 & 1.19 & 15 & 1.16 & 2.05 \\
\hline \multirow[t]{2}{*}{ Letrosol start day of stimulation } & 0 & 0 & 0 & 0.77 & 5 & 0.86 & 1.41 \\
\hline & 0 & 0 & 0 & 0.77 & 17 & 0.76 & 1.38 \\
\hline \multirow[t]{2}{*}{ Clomiphen dose (mg/day) } & 0 & 0 & 59.87 & 100 & 150 & 57.97 & 45.82 \\
\hline & 0 & 0 & 100 & 100 & 150 & 60.19 & 45.54 \\
\hline \multirow[t]{2}{*}{ Clomiphen duration } & 0 & 0 & 3 & 5 & 12 & 2.94 & 2.33 \\
\hline & 0 & 0 & 5 & 5 & 15 & 3.07 & 2.36 \\
\hline \multirow[t]{2}{*}{ Clomiphen Start day of stimulation } & 0 & 0 & 2.08 & 3 & 6 & 2 & 1.66 \\
\hline & 0 & 0 & 2.08 & 3 & 18 & 2.09 & 1.7 \\
\hline \multirow[t]{2}{*}{ Summary of foliculogenesis-duration of stimulation } & 1 & 9 & 10.23 & 11 & 25 & 10.41 & 2.59 \\
\hline & 1 & 9 & 10.23 & 11 & 31 & 10.2 & 2.63 \\
\hline \multirow[t]{2}{*}{ Fostimon dose IU } & 0 & 0 & 0 & 77.03 & 337.5 & 91.4 & 23.39 \\
\hline & 0 & 0 & 0 & 77.03 & 247.5 & 74.61 & 18.54 \\
\hline \multirow[t]{2}{*}{ Gonal.F dose IU } & 0 & 0 & 0 & 0 & 3750 & 60.96 & 26.71 \\
\hline & 0 & 0 & 0 & 0 & 6847 & 60.2 & 23.93 \\
\hline \multirow[t]{2}{*}{ Menopour Dose IU } & 0 & 0 & 0 & 0 & 2700 & 10.65 & 125.74 \\
\hline & 0 & 0 & 0 & 0 & 3300 & 9.74 & 104.92 \\
\hline \multirow[t]{2}{*}{ HMG dose IU } & 0 & 0 & 0 & 150 & 2625 & 116.59 & 221.67 \\
\hline & 0 & 0 & 0 & 150 & 3300 & 125.45 & 230.94 \\
\hline \multirow[t]{2}{*}{ Merional dose IU } & 0 & 0 & 0 & 0 & 3900 & 66.25 & 295.78 \\
\hline & 0 & 0 & 0 & 0 & 9075 & 44.76 & 202.57 \\
\hline \multirow[t]{2}{*}{ Hemogon dose IU } & 0 & 0 & 0 & 0 & 1200 & 3.09 & 44.01 \\
\hline & 0 & 0 & 0 & 0 & 3000 & 4.95 & 55.65 \\
\hline \multirow[t]{2}{*}{ Menogan dose IU } & 0 & 0 & 0 & 0 & 3450 & 46.63 & 195.23 \\
\hline & 0 & 0 & 0 & 0 & 3600 & 44.2 & 163.25 \\
\hline \multirow[t]{2}{*}{ Bravelle dose IU } & 0 & 0 & 0 & 0 & 1050 & 7.93 & 57.94 \\
\hline & 0 & 0 & 0 & 0 & 2100 & 9.55 & 77.96 \\
\hline \multirow[t]{2}{*}{ Gonadotropin total dose } & 0 & 225 & 375 & 450 & 4500 & 453.57 & 502.6 \\
\hline & 0 & 225 & 375 & 450 & 4424 & 411.52 & 410.99 \\
\hline Esteradiol dose & 0 & 0 & 0 & 0 & 6 & 0.33 & 0.99 \\
\hline & 0 & 0 & 0 & 0 & 8 & 0.42 & 1.16 \\
\hline Esteradiol duration & 0 & 0 & 0 & 0 & 15 & 0.25 & 0.95 \\
\hline & 0 & 0 & 0 & 0 & 14 & 0.3 & 0.96 \\
\hline No of dominate follicle at HCG day_17 & 0 & 1 & 1.65 & 2 & 10 & 1.81 & 1.46 \\
\hline & 0 & 1 & 1.65 & 2 & 11 & 1.61 & 1.38 \\
\hline No of dominate follicle at HCG day_18 & 0 & 0 & 0.95 & 1 & 7 & 0.97 & 0.95 \\
\hline & 0 & 0 & 0.94 & 1 & 9 & 0.92 & 0.87 \\
\hline
\end{tabular}


Table 9 (continued)

\begin{tabular}{|c|c|c|c|c|c|c|c|}
\hline Feature name & Min & First Q & Median & Third Q & Max & Mean & SD \\
\hline \multirow[t]{2}{*}{ HCG day endometrium (endometrial thickness) } & 4.3 & 8 & 8.51 & 9 & 19 & 8.67 & 1.47 \\
\hline & 1 & 8 & 8.52 & 9 & 18 & 8.48 & 1.51 \\
\hline \multirow[t]{2}{*}{ Age_y } & 20 & 30 & 33 & 35 & 72 & 32.82 & 4.7 \\
\hline & 20 & 30 & 33 & 36 & 80 & 33.35 & 4.88 \\
\hline \multirow[t]{2}{*}{ Family demographic information (Education_y) } & 1 & 2 & 3 & 4 & 7 & 3.38 & 1.23 \\
\hline & 1 & 2 & 3 & 4 & 7 & 3.31 & 1.25 \\
\hline \multirow[t]{2}{*}{ Specimen characteristics-abstinence } & 0.41 & 3 & 4 & 4.59 & 20 & 4.42 & 1.79 \\
\hline & 0.41 & 3 & 4 & 4.59 & 20 & 4.43 & 1.89 \\
\hline \multirow[t]{2}{*}{ Specimen characteristics-volume (normal range $2.7 \mathrm{~mL}$ ) } & 0.1 & 2 & 3.2 & 4 & 9.5 & 3.29 & 1.56 \\
\hline & 0.1 & 2 & 3 & 4 & 10.5 & 3.23 & 1.54 \\
\hline \multirow{2}{*}{ Specimen characteristics-PH } & 6 & 7.8 & 7.8 & 7.8 & 8.5 & 7.8 & 0.11 \\
\hline & 6 & 7.8 & 7.8 & 7.8 & 85 & 7.8 & 0.10 \\
\hline \multirow[t]{2}{*}{ Specimen characteristics liquefaction time (normal range15-30 min) } & 20 & 20 & 20 & 30 & 60 & 26.10 & 8.60 \\
\hline & 15 & 20 & 20 & 25.53 & 60 & 25.34 & 8.48 \\
\hline \multirow[t]{2}{*}{ Sperm concentration- total sperm count } & 10 & 104 & 170.1 & 202.72 & 399 & 165.46 & 84.57 \\
\hline & 10 & 92.5 & 168 & 204 & 400 & 163.60 & 88.18 \\
\hline \multirow[t]{2}{*}{ Sperm motility-total motility (normal range 50) } & 10 & 40 & 57.3 & 72.22 & 98.8 & 55.94 & 19.87 \\
\hline & 10 & 37.6 & 55.9 & 72.22 & 100 & 54.51 & 21.10 \\
\hline \multirow[t]{2}{*}{ Total motile sperm count } & 1.14 & 41.06 & 86.70 & 132.90 & 354.88 & 95.29 & 64.21 \\
\hline & 1 & 35.52 & 81.55 & 132.90 & 370.11 & 93.71 & 69.08 \\
\hline \multirow[t]{2}{*}{ Sperm motility-shaking grade } & 0 & 0 & 0 & 0 & 5 & 0.03 & 0.36 \\
\hline & 0 & 0 & 0 & 0 & 15 & 0.03 & 0.48 \\
\hline \multirow[t]{2}{*}{ Sperm motility-grade I- } & 0 & 0 & 0 & 0 & 19 & 0.12 & 1.14 \\
\hline & 0 & 0 & 0 & 0 & 34.3 & 0.12 & 1.16 \\
\hline \multirow[t]{2}{*}{ Sperm motility-grade I (grade d) } & 0 & 0 & 3.6 & 8.2 & 37.3 & 5.3 & 5.92 \\
\hline & 0 & 0 & 3.6 & 8.2 & 46.4 & 5.40 & 6.18 \\
\hline \multirow[t]{2}{*}{ Sperm motility Grade II- } & 0 & 0 & 0 & 0 & 50.3 & 0.27 & 3.04 \\
\hline & 0 & 0 & 0 & 0 & 57.6 & 0.21 & 2.57 \\
\hline \multirow[t]{2}{*}{ Sperm motility Grade II (grade c) } & 0 & 10.27 & 26.7 & 36.8 & 64.7 & 24.11 & 16.26 \\
\hline & 0 & 11.9 & 26.4 & 36.7 & 70.0 & 24.25 & 15.97 \\
\hline \multirow[t]{2}{*}{ Sperm motility Grade II+ } & 0 & 0 & 0 & 0 & 23.2 & 0.01 & 0.58 \\
\hline & 0 & 0 & 0 & 0 & 37.3 & 0 & 0.38 \\
\hline \multirow[t]{2}{*}{ Sperm motility Grade III $(a+b)$} & 0 & 2.7 & 9.8 & 18.6 & 48.9 & 11.96 & 10.76 \\
\hline & 0 & 3.3 & 9.8 & 17.8 & 49.8 & 11.96 & 10.82 \\
\hline \multirow[t]{2}{*}{ Sperm morphology-normal morphology (normal range 30) } & 0 & 4 & 5.62 & 7 & 20 & 5.72 & 2.99 \\
\hline & 0 & 4 & 5.62 & 7 & 28 & 5.6 & 3.1 \\
\hline \multirow[t]{2}{*}{ Sperm morphology-abnormal morphology (normal range 70) } & 80 & 93 & 94.38 & 96 & 100 & 94.27 & 2.99 \\
\hline & 72 & 93 & 94.38 & 96 & 133 & 94.4 & 3.13 \\
\hline \multirow[t]{2}{*}{ Sperm morphology-amorph } & 0 & 69 & 76 & 80 & 92 & 68.59 & 22.61 \\
\hline & 0 & 68.66 & 75 & 80 & 96 & 68.68 & 21.7 \\
\hline Sperm morphology-double head & 0 & 0 & 0 & 1.02 & 8 & 0.85 & 1.20 \\
\hline & 0 & 0 & 1 & 2 & 11 & 1.02 & 1.36 \\
\hline Sperm morphology-giant head & 0 & 1 & 2 & 4 & 20 & 2.40 & 2.51 \\
\hline & 0 & 1 & 2 & 4 & 25 & 2.53 & 2.61 \\
\hline Sperm morphology-pin head & 0 & 1 & 2 & 4 & 30 & 2.95 & 3.47 \\
\hline & 0 & 1 & 2 & 4 & 30 & 3.14 & 3.37 \\
\hline Sperm morphology-round head & 0 & 1 & 2 & 4 & 20 & 2.85 & 2.92 \\
\hline & 0 & 1 & 2 & 4 & 20 & 2.66 & 2.69 \\
\hline Sperm morphology-double tail & 0 & 0 & 0 & 0 & 4 & 0.14 & 0.47 \\
\hline
\end{tabular}


Table 9 (continued)

\begin{tabular}{|c|c|c|c|c|c|c|c|}
\hline Feature name & Min & First Q & Median & Third Q & Max & Mean & SD \\
\hline & 0 & 0 & 0 & 0 & 10 & 0.19 & 0.63 \\
\hline \multirow[t]{2}{*}{ Sperm morphology-coiled tail } & 0 & 1 & 3 & 5 & 25 & 3.79 & 3.48 \\
\hline & 0 & 2 & 3 & 6 & 28 & 4.17 & 3.84 \\
\hline \multirow[t]{2}{*}{ Sperm morphology-short tail } & 0 & 1 & 1 & 2.31 & 24 & 1.90 & 2.37 \\
\hline & 0 & 1 & 1 & 3 & 28 & 2.20 & 2.75 \\
\hline \multirow[t]{2}{*}{ Sperm morphology-cytoplasmic droplet } & 1 & 4 & 7 & 14 & 20 & 8.08 & 4.94 \\
\hline & 1 & 4 & 7 & 12 & 30 & 8.12 & 4.85 \\
\hline \multirow[t]{2}{*}{ Other tests-germinal cell } & 0 & 1 & 2 & 3 & 15 & 2.14 & 1.87 \\
\hline & 0 & 1 & 2 & 3 & 21 & 2.20 & 1.79 \\
\hline \multirow[t]{2}{*}{ Therapeutic duration of liquefaction (min) } & 5 & 15 & 16.13 & 16.13 & 145 & 16.16 & 5.70 \\
\hline & 5 & 15 & 16.13 & 16.13 & 200 & 16.15 & 5.22 \\
\hline \multirow[t]{2}{*}{ Therapeutic volume } & 0.5 & 2.5 & 3.59 & 4 & 13 & 3.6 & 1.64 \\
\hline & 0.01 & 2.5 & 3.5 & 4 & 13 & 3.54 & 1.66 \\
\hline \multirow[t]{2}{*}{ Therapeutic PH } & 5 & 7.76 & 7.8 & 7.8 & 7.9 & 7.77 & 0.09 \\
\hline & 0.33 & 7.76 & 7.8 & 7.8 & 10 & 7.76 & 0.19 \\
\hline \multirow[t]{2}{*}{ Before processing motility } & 0.04 & 0.3 & 0.37 & 0.45 & 0.92 & 0.38 & 0.14 \\
\hline & 0.01 & 0.28 & 0.37 & 0.45 & 0.98 & 0.37 & 0.14 \\
\hline \multirow[t]{2}{*}{ Before processing sperm concentration } & 4 & 45 & 54.62 & 68 & 110 & 55.57 & 18.7 \\
\hline & 1 & 42 & 54.62 & 68 & 110 & 54.15 & 20.23 \\
\hline \multirow[t]{2}{*}{ After processing motility } & 0.2 & 0.96 & 0.98 & 1 & 1 & 0.97 & 0.06 \\
\hline & 0.02 & 0.96 & 0.98 & 1 & 1 & 0.96 & 0.09 \\
\hline \multirow[t]{2}{*}{ Post wash total motile sperm count } & 1 & 127.4 & 209.88 & 264.87 & 460 & 213.81 & 27.49 \\
\hline & 0.16 & 102.9 & 195 & 252 & 450 & 198.81 & 35.09 \\
\hline \multirow[t]{2}{*}{ After processing sperm concentration } & 2 & 50 & 60.7 & 72 & 120 & 60.82 & 21.94 \\
\hline & 1 & 42 & 60.7 & 72 & 120 & 57.23 & 24.87 \\
\hline
\end{tabular}

* Statistical description of numeric variables: Each row is divided into two parts in which the top row is related to the positive class and the bottom row represents the negative class

Received: 8 December 2019 Accepted: 3 December 2020

Published online: 02 January 2021

\section{References}

1. Practice Committee of the American Society for Reproductive Medicine. Definitions of infertility and recurrent pregnancy loss: a committee opinion. Fertil Steril. 2013;99(1):63.

2. Borght $M$, Wyns $C$. Fertility and infertility: definition and epidemiology Clin Biochem. 2018;62:2-10.

3. Milewska AJ, et al. Prediction of infertility treatment outcomes using classification trees. Stud Log Gramm Rhetoric. 2016;47(1):7-19.

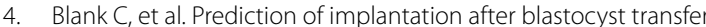
in in vitro fertilization: a machine-learning perspective. Fertil Steril. 2019:111(2):318-26.

5. Patil AS. A review of soft computing used in assisted reproductive techniques (ART). Int J Eng Trends Appl (IJETA). 2015;2(3):88-93.

6. Bahadur $\mathrm{G}$, et al. First line fertility treatment strategies regarding IUI and IVF require clinical evidence. Hum Reprod. 2016;31(6):1141-6.

7. Ombelet W, Puttemans P, Bosmans E. Intrauterine insemination: a firststep procedure in the algorithm of male subfertility treatment. Hum Reprod. 1995;10:90-102.

8. Deo RC. Machine learning in medicine. Circulation. 2015;132(20):1920-30.

9. Milewska AJ, et al. Analyzing outcomes of intrauterine insemination treatment by application of cluster analysis or kohonen neural networks. Stud Log Gramm Rhetoric. 2013:35(1):7-25.

10. Kooptiwoot S, Salam MA. IUI mining: human expert guidance of information theoretic network approach. Soft Comput. 2006;10(4):369-73.
11. Ghaffari $F$, et al. Evaluating the effective factors in pregnancy after intrauterine insemination: a retrospective study. Int J Fertil Steril. 2015;9(3):300.

12. Steures $P$, et al. Prediction of an ongoing pregnancy after intrauterine insemination. Fertil Steril. 2004;82(1):45-51.

13. Goldman $\mathrm{RH}$, et al. Patient-specific predictions of outcome after gonadotropin ovulation induction/intrauterine insemination. Fertil Steril. 2014;101(6):1649-55

14. Marshburn PB, et al. Spermatozoal characteristics from fresh and frozen donor semen and their correlation with fertility outcome after intrauterine insemination. Fertil Steril. 1992;58(1):179-86.

15. Moro F, et al. Anti-Müllerian hormone concentrations and antral follicle counts for the prediction of pregnancy outcomes after intrauterine insemination. Int J Gynecol Obstet. 2016;133(1):64-8.

16. Lemmens $L$, et al. Predictive value of sperm morphology and progressively motile sperm count for pregnancy outcomes in intrauterine insemination. Fertil Steril. 2016;105(6):1462-8.

17. Arslan $M$, et al. Predictive value of the hemizona assay for pregnancy outcome in patients undergoing controlled ovarian hyperstimulation with intrauterine insemination. Fertil Steril. 2006;85(6):1697-707.

18. Florio $P$, et al. Evaluation of endometrial activin A secretion for prediction of pregnancy after intrauterine insemination. Fertil Steril. 2010;93(7):2316-20.

19. Shah S, Kusiak A. Cancer gene search with data-mining and genetic algorithms. Comput Biol Med. 2007;37(2):251-61.

20. Kaya A. Cascaded classifiers and stacking methods for classification of pulmonary nodule characteristics. Comput Methods Programs Biomed. 2018;166:77-89. 
21. Wang SQ, Yang J, Chou KC. Using stacked generalization to predict membrane protein types based on pseudo-amino acid composition. J Theor Biol. 2006;242(4):941-6.

22. Tocci A, Lucchini C. WHO reference values for human semen. Hum Reprod Update. 2010;16(5):559-559.

23. Zhang S, Zhang C, Yang Q. Data preparation for data mining. Appl Artif Intell. 2003;17(5-6):375-81.

24. Han J, Pei J, Kamber M. Data mining: concepts and techniques. Amsterdam: Elsevier; 2011

25. Liu FT, Ting KM, Zhou ZH, Isolation forest, in 2008 Eighth IEEE international conference on data mining. 2008, IEEE. p. 413-422

26. Breiman L. Random forests. Mach Learn. 2001;45:5-32.

27. Diykh M, Li Y, Abdulla S. EEG sleep stages identification based on weighted undirected complex networks. Comput Methods Programs Biomed. 2020;184:105116.

28. Bavelas A. A mathematical model for group structure, human organization. Appl Anthropol. 1948;7(3):16-30.

29. Wolpert DH. Stacked generalization. Neural Netw. 1992;5(2):241-59.

30. Güneş F, Wolfinger R, Tan PY. Stacked ensemble models for improved prediction accuracy. in Static Anal. Symp. 2017.

31. Sperandei S. Understanding logistic regression analysis. Biochem Med. 2014;24(1):12-8.

32. Cortes C, Vapnik V. Support-vector network. Mach Learn. 1995;20:1-25.

33. Quinlan JR. Induction of decision trees. Mach Learn. 1986;1:81-106.

34. Zhu J, et al. Multi-class AdaBoost. Stat Interfere. 2009:2:349-60.

35. Ke, G., et al. Lightgbm: a highly efficient gradient boosting decision tree. in Advances in neural information processing systems. 2017.

36. Gharehbaghi A, Linden M. A deep machine learning method for classifying cyclic time series of biological signals using time-growing neural network. IEEE Trans Neural Netw Learn Syst. 2018;29(9):4102-15.

37. Campana A, et al. Intrauterine insemination: evaluation of the results according to the woman's age, sperm quality, total sperm count per insemination and life table analysis. Hum Reprod. 1996;11(4):732-6.

38. Kuriya A, Agbo C, Dahan MH. Do pregnancy rates differ with intrauterine insemination when different combinations of semen analysis parameters are abnormal? J Turk German Gynecol Assoc. 2018;19(2):57.

39. Zhang E, et al. Effect of sperm count on success of intrauterine insemination in couples diagnosed with male factor infertility. Materia Socio-Medica. 2014;26(5):321.

40. Ombelet $W$, et al. Semen quality and intrauterine insemination. Reprod BioMed Online. 2003;7(4):485-92.

41. Dickey RP, et al. Comparison of the sperm quality necessary for successful intrauterine insemination with World Health Organization threshold values for normal sperm. Fertil Steril. 1999:71(4):684-9.
42. Duran HE, et al. Sperm DNA quality predicts intrauterine insemination outcome: a prospective cohort study. Hum Reprod. 2002:17(12):3122-8.

43. Muriel $L$, et al. Value of the sperm chromatin dispersion test in predicting pregnancy outcome in intrauterine insemination: a blind prospective study. Hum Reprod. 2006;21(3):738-44.

44. Thijssen A, et al. Predictive factors influencing pregnancy rates after intrauterine insemination with frozen donor semen: a prospective cohort study. Reprod Biomed Online. 2017;34(6):590-7.

45. Merviel $P$, et al. Predictive factors for pregnancy after intrauterine insemination (IUI): An analysis of 1038 cycles and a review of the literature. Fertil Steril. 2010;93(1):79-88.

46. Erdem $A$, et al. Factors affecting live birth rate in intrauterine insemination cycles with recombinant gonadotrophin stimulation. Reprod Biomed Online. 2008;17(2):199-206.

47. Kamath MS, et al. Predictive factors for pregnancy after intrauterine insemination: a prospective study of factors affecting outcome. Hum Reprod Sci. 2010;3(3):129.

48. Licht RS, Handel L, Sigman M. Site of semen collection and its effect on semen analysis parameters. Fertil Steril. 2008;89(2):395-7.

49. Francavilla F, et al. Effect of sperm morphology and motile sperm count on outcome of intrauterine insemination in oligozoospermia and/or asthenozoospermia. Fertil Steril. 1990;53(5):892-7.

50. Luco SM, et al. The evaluation of pre and post processing semen analysis parameters at the time of intrauterine insemination in couples diagnosed with male factor infertility and pregnancy rates based on stimulation agent. A retrospective cohort study. Eur J Obstet Gynecol Reprod Bio Endocrinol. 2014;179:159-62.

51. Blank C, et al. Prediction of implantation after blastocyst transfer in in vitro fertilization: a machine-learning perspective. Fertil Steril. 2019:111(2):318-26.

52. Nuojua-Huttunen $\mathrm{S}$, et al. Intrauterine insemination treatment in subfertility: an analysis of factors affecting outcome. Hum Reprod. 1999;14(3):698-703.

53. Liu W, et al. Comparing the pregnancy rates of one versus two intrauterine inseminations (IUIs) in male factor and idiopathic infertility. J Assist Reprod Genet. 2006;23(2):75-9.

\section{Publisher's Note}

Springer Nature remains neutral with regard to jurisdictional claims in published maps and institutional affiliations.
Ready to submit your research? Choose BMC and benefit from:

- fast, convenient online submission

- thorough peer review by experienced researchers in your field

- rapid publication on acceptance

- support for research data, including large and complex data types

- gold Open Access which fosters wider collaboration and increased citations

- maximum visibility for your research: over $100 \mathrm{M}$ website views per year

At BMC, research is always in progress.

Learn more biomedcentral.com/submissions 